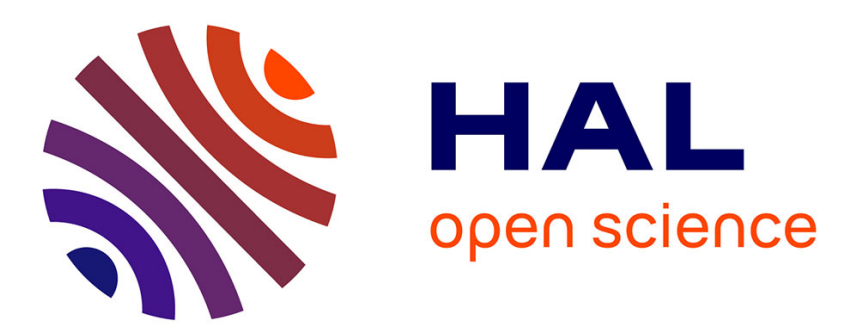

\title{
Stiffness modeling for perfect and non-perfect parallel manipulators under internal and external loadings
}

\author{
Alexandr Klimchik, Damien Chablat, Anatol Pashkevich
}

\section{To cite this version:}

Alexandr Klimchik, Damien Chablat, Anatol Pashkevich. Stiffness modeling for perfect and nonperfect parallel manipulators under internal and external loadings. Mechanism and Machine Theory, 2014, 79, pp.1-28. 10.1016/j.mechmachtheory.2014.04.002 . hal-01065144

\section{HAL Id: hal-01065144 \\ https://hal.science/hal-01065144}

Submitted on 18 Sep 2014

HAL is a multi-disciplinary open access archive for the deposit and dissemination of scientific research documents, whether they are published or not. The documents may come from teaching and research institutions in France or abroad, or from public or private research centers.
L'archive ouverte pluridisciplinaire HAL, est destinée au dépôt et à la diffusion de documents scientifiques de niveau recherche, publiés ou non, émanant des établissements d'enseignement et de recherche français ou étrangers, des laboratoires publics ou privés. 


\title{
Stiffness modeling for perfect and non-perfect parallel manipulators under internal and external loadings
}

\author{
Alexandr Klimchik ${ }^{\mathrm{a}, \mathrm{b}},{ }_{\text {, Damien Chablat }}^{\mathrm{b}, \mathrm{c}}$, Anatol Pashkevich ${ }^{\mathrm{a}, \mathrm{b}}$, \\ ${ }^{a}$ Ecole des Mines de Nantes, 4 rue Alfred-Kastler, Nantes 44307, France \\ ${ }^{b}$ Institut de Recherches en Communications et en Cybernétique de Nantes, UMR CNRS 6597, 1 rue de la Noe, 44321 Nantes, France \\ ${ }^{c}$ Centre national de la recherche scientifique (CNRS), France
}

\begin{abstract}
The paper presents an advanced stiffness modeling technique for perfect and non-perfect parallel manipulators under internal and external loadings. Particular attention is paid to the manipulators composed of non-perfect serial chains, whose geometrical parameters differ from the nominal ones and do not allow to assemble manipulator without internal stresses that considerably affect the stiffness properties and also change the end-effector location. In contrast to other works, several types of loadings are considered simultaneously: an external force applied to the end-effector, internal loadings generated by the assembling of non-perfect serial chains and external loadings applied to the intermediate points (auxiliary loading due to the gravity forces and relevant compensator mechanisms, etc.). For this type of manipulators, a non-linear stiffness modeling technique is proposed that allows to take into account inaccuracy in the chains and to aggregate their stiffness models for the case of both small and large deflections. Advantages of the developed technique and its ability to compute and compensate the compliance errors caused by the considered factors are illustrated by an example that deals with parallel manipulators of the Orthoglide family.
\end{abstract}

Keywords:

Non-linear stiffness modeling, parallel manipulators, compliance errors, non-perfect manipulators.

\section{Introduction}

Stiffness modeling of robotic manipulator is one of the important issues that allows user to evaluate its compatibility for certain tasks. It becomes especially critical for parallel manipulators, for which robot manufactures tend to decrease moving masses via reducing the link cross-sections. The latter improves the robot dynamics but obviously leads to the deterioration of the manipulator resistance with respect to the external forces. In some application areas where high precision is required (robotic based machining, etc.), the stiffness model is in the core of the relevant compliance errors compensation technique and its precision defines the final product quality. For these reasons, the problem of the manipulator stiffness modeling permanently attracts attention of the research community.

Currently, there are a number of works that contains essential results on the manipulator modeling under the external loading. Some of them take into account very specific features caused by the influence of the second order derivatives [1,2], impact of passive joints [3,4] or effect of internal constraints [5,6]. Other works concentrate the problem of stiffness analysis for the manipulators with internal preloading or antagonistic actuating [7-10]. A number of authors address more general problems arising in stiffness analysis of serial and parallel manipulators [11-14]. There are also quite a few works focusing on particular architectures [15-17]. However, some important issues have remained beyond the scope of research activities, they include influence of internal loadings caused by geometrical errors in over-constrained parallel manipulators and impact of auxiliary loadings applied to the intermediate points (different from the end-effector). This paper contributes to the enhancement of the existing stiffness modeling methods by focusing on the above mentioned issues.

The remainder of this paper is organized as follows. Section 2 presents detailed analysis of related works on the stiffness modeling of robotic manipulator. Section 3 deals with the stiffness modeling of serial chain and proposes equilibrium equations and a numerical algorithm for computing of the loaded static equilibrium for the manipulator under internal and external lading as well as equations for computing corresponding stiffness matrix and stresses in virtual joints. Section 5 focuses on the stiffness modeling of parallel manipulators and presents aggregation techniques for the manipulators with perfect and non-perfect serial chains under internal and external loadings. Section 6 contains a set of illustrative examples that demonstrate the advantages of developed technique. Section 7 presents some discussion concerning limitations and possible extensions of the developed method. And finally, Section 8 summarizes the main results and contributions.

\footnotetext{
${ }^{1}$ Corresponding author. Tel. +33 2518583 17; fax. +33 2518583 49; E-mail address: alexandr.klimchik@ @ines-nantes.fr (A. Klimchik).
} 


\section{Related works on the stiffness modeling of robotic manipulator}

To ensure efficient compensation of the compliance errors in the robot-based machining, an appropriate stiffness model which is able to take into account both changes in manipulator configuration and influence of the external forces/torques is required. This section presents an analytical review of existing approaches in the stiffness modeling of robotic manipulators of both serial and parallel architectures, with special attention to the virtual joint method providing a reasonable compromise between the model accuracy and computational efficiency. In addition, a special emphasis is given to the stiffness modeling in the loaded mode, which is essential for the considered application area.

\subsection{Problem of stiffness modeling and existing approaches}

Existing approaches. Similar to general structural mechanics, the robot stiffness characterizes the manipulator resistance to the deformations caused by an external force or torque applied to the end-effector $[18,19]$. Numerically, this property is usually defined through the stiffness matrix $\mathbf{K}$, which is incorporated in a linear relation between the translational/rotational displacement and static forces/torques causing this transition (assuming that all of them are small enough). The inverse of $\mathbf{K}$ is usually called the compliance matrix and is denoted as $\mathbf{k}$. As it follows from related works, for conservative systems $\mathbf{K}$ is a $6 \times 6$ semi-definite non-negative symmetrical matrix but its structure may be non-diagonal to represent the coupling between the translation and rotation [12]. However, in general, for non-conservative systems and/or some special parameterizations of end-effector location, in the loaded mode the stiffness matrix may be asymmetrical ${ }^{2}$.

In stiffness modeling of robotic manipulator, because of some specificity, there are some peculiarities in terminology. In particular, the matrix $\mathbf{K}$ is usually referred to as the "Cartesian Stiffness Matrix" $\mathbf{K}_{\mathrm{C}}$ and it is distinguished from the "Joint-Space Stiffness Matrix" $\mathbf{K}_{\theta}$ that describes the relationship between the static forces/torques and corresponding deflections in the joints [28]. Both of these stiffness matrices can be mapped to each other using the Conservative Congruency Transformation [29-31], which is trivial if the external (or internal) loading is negligible. In this case, the transformation is entirely defined by the corresponding Jacobian matrix. However, if the loading is essential, it is described by a more complicated equation that includes both the Jacobian as well as the Jacobian derivatives and the loading vector [1,5]. Other specific cases, where the above transformation is non-trivial (non-linear or even singular), are related to manipulators with passive joints and over-constrained parallel architectures $[3,4,10]$. Since this work contributes to both problems, these issues will be considered in more detail below.

In the most general sense, existing approaches to the manipulator stiffness modeling may be roughly divided into three main groups: (i) the Finite Elements Analysis (FEA), (ii) the Matrix Structural Analysis (MSA), and (iii) the Virtual Joint Modeling method (VJM). Their advantages and disadvantages are briefly presented below, with special emphasis to the computational complexity and accuracy.

Finite Element Analysis method (FEA). Its basic idea is to decompose the physical model of the mechanical structure on a number of rather small (finite) elements and to introduce compliant relations between adjacent nods described by relevant stiffness matrices. These finite elements have a standard shape (pyramids, cubes, etc.) for which the stiffness matrix can be computed analytically. Using this discretization, the static equilibrium equations for each node are derived, and they are aggregated in a global matrix expression defining relations between the applied force/torque and node deflections. Then, the obtained matrix of rather large size is inverted and is used to obtain the desired stiffness matrix by simple extraction of proper elements. In the modern CAD-environment, the above process is highly automated and is integrated with 3D-modeling of mechanical structures and mechanisms. In particular, the decomposition into a set of finite elements (so called meshing) usually needs definition of the discretization step and the mesh type only. The latter can be either linear or parabolic, which correspond to pyramids with 4 and 10 nodes respectively (with either 6 or 12 compliance relations). Then (for this finite element model), the CAD-based tools provides both numerical data and convenient visualization defining the deflections vectors for each nodes and potential dangerous areas with high stresses.

An evident advantage of the FEA-modeling is its high accuracy that is limited by the discretization step only. For robotic application it is very attractive, since the links/joints are modeled with its true dimension and shape [16,32,33]. However, while increasing of the number of finite elements, the problem of limited computer memory and the difficulty of the high-dimension matrix inversion become more and more critical. Besides the high computational efforts, this matrix inversion generates numerous accumulative round-off errors, which reduce accuracy. In robotics, this causes rather high computational expenses for the repeated re-meshing and re-computing, so in this area the FEA method can be applied for the links stiffness matrix identification [34] that is further used in the frame of the VJM technique. Such combination allows us to use the advantages of the FEA while avoiding intensive computations for different manipulator configurations.

\footnotetext{
${ }^{2}$ In robotic literature, there is rather intensive discussions concerning the symmetry of the stiffness matrix in the loaded mode [20-27]. But in this work, due to the adopted assumptions, the stiffness matrix is certainly symmetrical.
} 
Matrix Structural Analysis method (MSA). This method incorporates the main ideas of the FEA but operates with rather large compliant elements such as beams, arcs, cables, etc. [35]. This obviously leads to the reduction of the computational expenses and, in some cases, allows us to obtain an analytical stiffness matrix for the specific task. Similar to the FEA-modeling the MSA method gives forces/torques and displacements for each node, but here it has a clear physical interpretation (manipulator active or passive joint), which can be useful for some tasks [14,36]. For parallel robots, this method has been developed in works [37,38], where a general technique for stiffness modeling of the manipulator with rigid/flexible links and passive joints was proposed. It has been illustrated by stiffness analysis of parallel manipulator of Delta architecture where the links were approximated by regular beams. The latter causes some doubts in the model accuracy compared to the combination of the FEA and VJM techniques that are being developed here. Besides, this result was obtained under the assumption that the external forces/torques are relatively small (i.e. for the unloaded mode), and it is unlikely that such approach can be enhanced to take into account particularities of manipulator behavior in loaded mode. In addition, here there exists a problem of the stiffness matrix computation for the manipulator singular configurations.

From a computational point of view, the MSA method is less complicated than the FEA-based one. In spite of the fact that the MSA still involves matrix operations of rather high-dimension, it gives a reasonable trade-off between the accuracy and computational time, provided that links approximations by the beam elements are realistic. It should be also noted that, in their general formulations, the FEA and MSA methods are closed: both of them interpret physical system as a set of nodes with mutual flexible connections. The main difference is that the MSA operates with true physical objects (like beams, arcs and others), while the FEA decomposes them into small finite elements. So, the MSA can be treated as a special case of the FEA that has already found its application in robotics.

From the other side, if each link is applied to the FEA-based stiffness matrix identification technique developed in [34], an advanced combination of the MSA and FEA that is suitable for the stiffness modeling of arbitrary parallel manipulators (with numerous internal loops) can be obtained. However, it is out of the scope of this work because of the above mentioned critical limitation, which makes the method applicable to the case of the unloaded mode only.

Virtual Joint Modeling method (VJM). The core of this method is an extension of the conventional rigid-body model of the robotic manipulator, where the links are treated as rigid but the joints are assumed to be compliant (in order to accumulate all types of existing flexibilities in the joints only). Geometrically, such approximation is equivalent to adding to the joints some auxiliary virtual joints (with embedded virtual springs). It is obvious that such lumped presentation of the manipulator stiffness (that in reality is distributed) essentially simplifies the model. So, at present it is the most popular stiffness analysis method in robotics. This method was first introduced by Salisbury [39] and Gosselin [40], who assumed that the main flexibility sources were concentrated in the actuator joints. The derived expression defining relation between the joint and Cartesian stiffness matrices (Conservative Congruency Transformation) became a basis for the manipulator stiffness analysis in many research works. Later, these results have been further developed in order to take into account some specific geometrical constrains $[5,41]$ and external loading [1,2]. Nevertheless, external loading is assumed small enough to detect any non-linear effects discovered in this work. Due to its computational simplicity, the VJM method has also been successfully applied to the analytical stiffness analysis of a translational parallel manipulator [42].

A key issue of this method is how to define the virtual spring parameters. At the beginning, it was assumed that each actuated joint is presented by a single one-dimensional virtual spring [11,24,43]. Further, to take into account the links flexibilities, the number of virtual joints was increased and in each actual actuated or passive joint several translational and rotational virtual springs were included [42]. The latest developments in this area operate with 6-dimensinal virtual springs identified using the FEA-based method [3,34]. This leads to essential increasing of the VJM method accuracy that becomes comparable with the accuracy of the FEA-based techniques, but with much lower computational expenses.

The only essential disadvantage of the VJM method is related to some difficulty in stiffness modeling of parallel manipulators. While for strictly parallel architectures, where there are only serial chains between the base and moving platforms, for architectures with internal loops (or with parallelogram-based links) the VJM-base stiffness analysis is rather complicated. Nevertheless, taking into account all advantages and disadvantages of the FEA, the MSA and the VJM techniques, the VJM method looks the most attractive in robotics.

Computational complexity. The main benchmark that allows us to evaluate the computational complexity of the above described methods is related to the computational efforts required for the matrix inversions inside of the corresponding algorithms. It should be stressed that the matrix inversion is the most time consuming operation for both linear and non-linear stiffness modeling. Generally, for the matrix inversion of size $n \times n$, it can be defined as $O\left(n^{3}\right)$ [44].

For the FEA method $n$ depends on the discretization step and the type of finite elements used. As it follows from a relevant study, in the case of the parabolic mesh (10 nodes and 12 connections per a finite element) the value of $n$ can be approximately computed as $n=30 v n_{L}$, where $n_{L}$ is the number of manipulator links, $v$ is the number of finite elements per link (it should be about $10^{3}$ to ensure desired precision). For the MSA method, the upper bound of the above matrix size $n=12 n_{d}$ can be computed 
via the node number $n_{d}$. Finally, for the VJM method, the size of the matrix to be inverted is $n=6+n_{q}$, where $n_{q}$ is total number of the degrees of freedom in the passive joints.

Using these formulas, it was estimated the stiffness model complexity for three types of manipulators (Stewart platform, Delta, Orthoglide). Relevant results are presented in Table 1. As it follows from them, the VJM method essentially overcomes FEA and MSA, so it will be used in this work as a preferable one. It is presented in more details below.

Table 1 Computational complexity of existing stiffness modeling methods

\begin{tabular}{cccc}
\hline & \multicolumn{3}{c}{ Manipulator architecture } \\
\cline { 2 - 4 } Stiffness modeling method & $\begin{array}{c}\text { Stewart Platform } \\
\text { (6 serial chains, each with } \\
2 \text { links and 5 passive joints) }\end{array}$ & $\begin{array}{c}\text { Delta } \\
\text { (3 serial chains, each with }\end{array}$ & $\begin{array}{c}\text { Orthoglide } \\
\text { (3 serial chains, each with } \\
5 \text { links and 4 passive joints) }\end{array}$ \\
\hline FEA & $\square 10^{16}\left(n_{L}=13\right)$ & $\square 10^{17}\left(n_{L}=19\right)$ & $\square 10^{17}\left(n_{L}=16\right)$ \\
MSA & $\square 10^{7}\left(n_{d}=5 \times 6\right)$ & $\square 10^{8}\left(n_{d}=11 \times 3\right)$ & $\square 10^{8}\left(n_{d}=13 \times 3\right)$ \\
VJM & $\square 10^{3}\left(n_{q}=5\right)$ & $\square 10^{3}\left(n_{q}=5\right)$ & $\square 10^{3}\left(n_{q}=4\right)$
\end{tabular}

$n_{L}, n_{d}, n_{q}$ are the number of links, node points and passive joints (in a single chain) respectively

\subsection{Virtual Joint Method in stiffness modeling of robots}

Since the VJM-based method is proved to be more computationally efficient while providing acceptable accuracy, let us consider it in more details. Taking into account some specificities of the considered application area, the main attention will be paid to the VJM applicability to the stiffness modeling of parallel manipulators (both under-constrained and over-constrained), considering the impact of the passive joint, evaluating the influence of the external and internal forces/torques as well as accuracy improvement of the stiffness model.

VJM method background. In the frame of this method, all types of compliance existing in a real manipulator (both distributed and lumped) are replaced by localized virtual springs located in its joints (Table 2). Example of VJM modeling is given in Figure 1, where both kinematic model of Stewart Platform and its VJM model are presented. Then, for this mechanism consisting of rigid links and compliance joints, the static equilibrium equations is derived and linearized in order to obtain Cartesian stiffness matrix, which in a general case depends on the manipulator posture (configuration) [10]. Usually it is assumed that the elastic deflections in virtual springs are relatively small and linearization is performed in the neighborhood of the equilibrium configuration corresponding to zero forces and torques (unloaded mode).

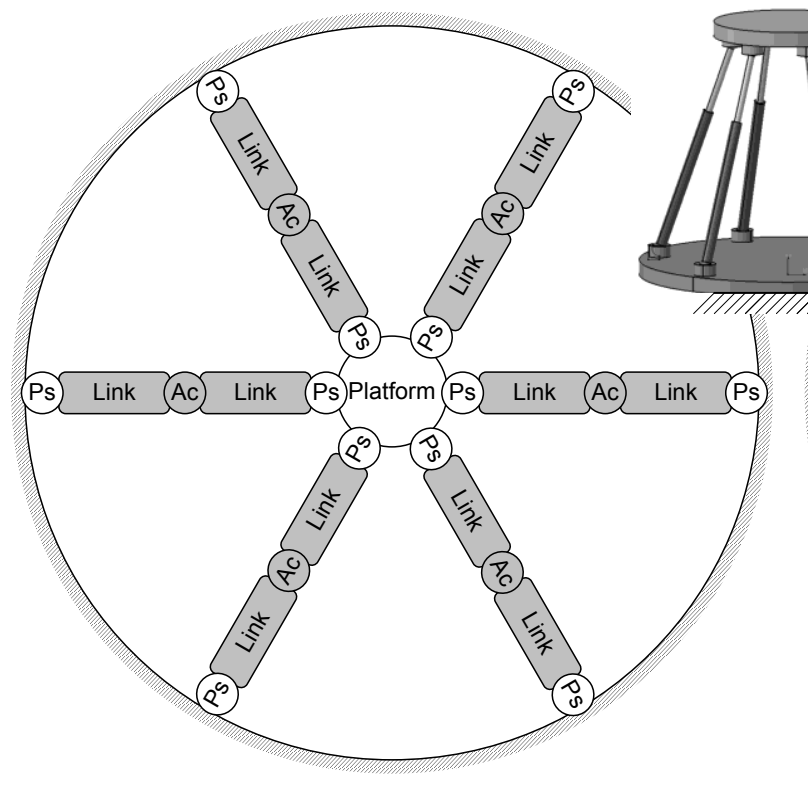

a) manipulator architecture

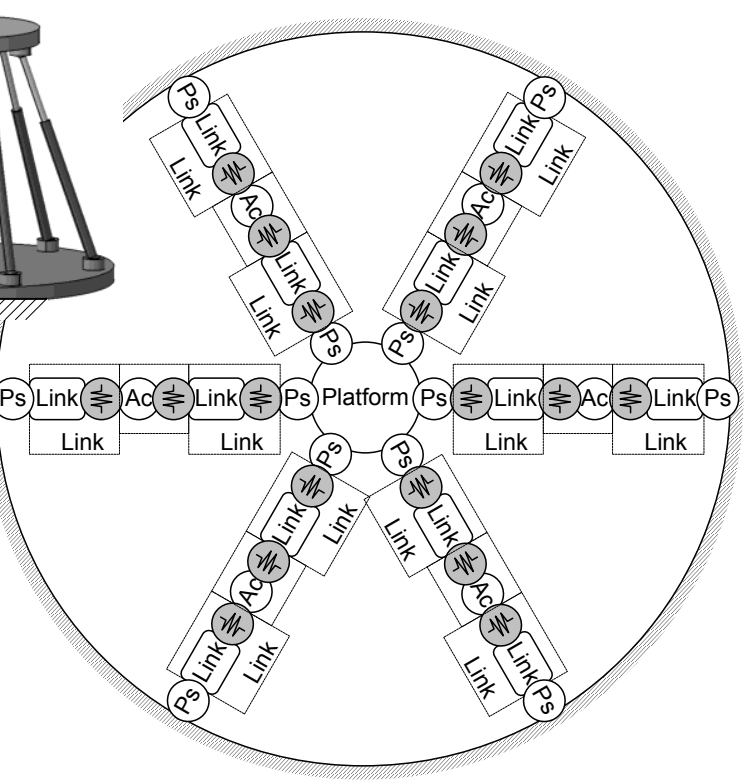

b) VJM model

Figure 1 Kinematic model of Stewart Platform and its VJM model 
Table 2 VJM-based modeling of manipulator components

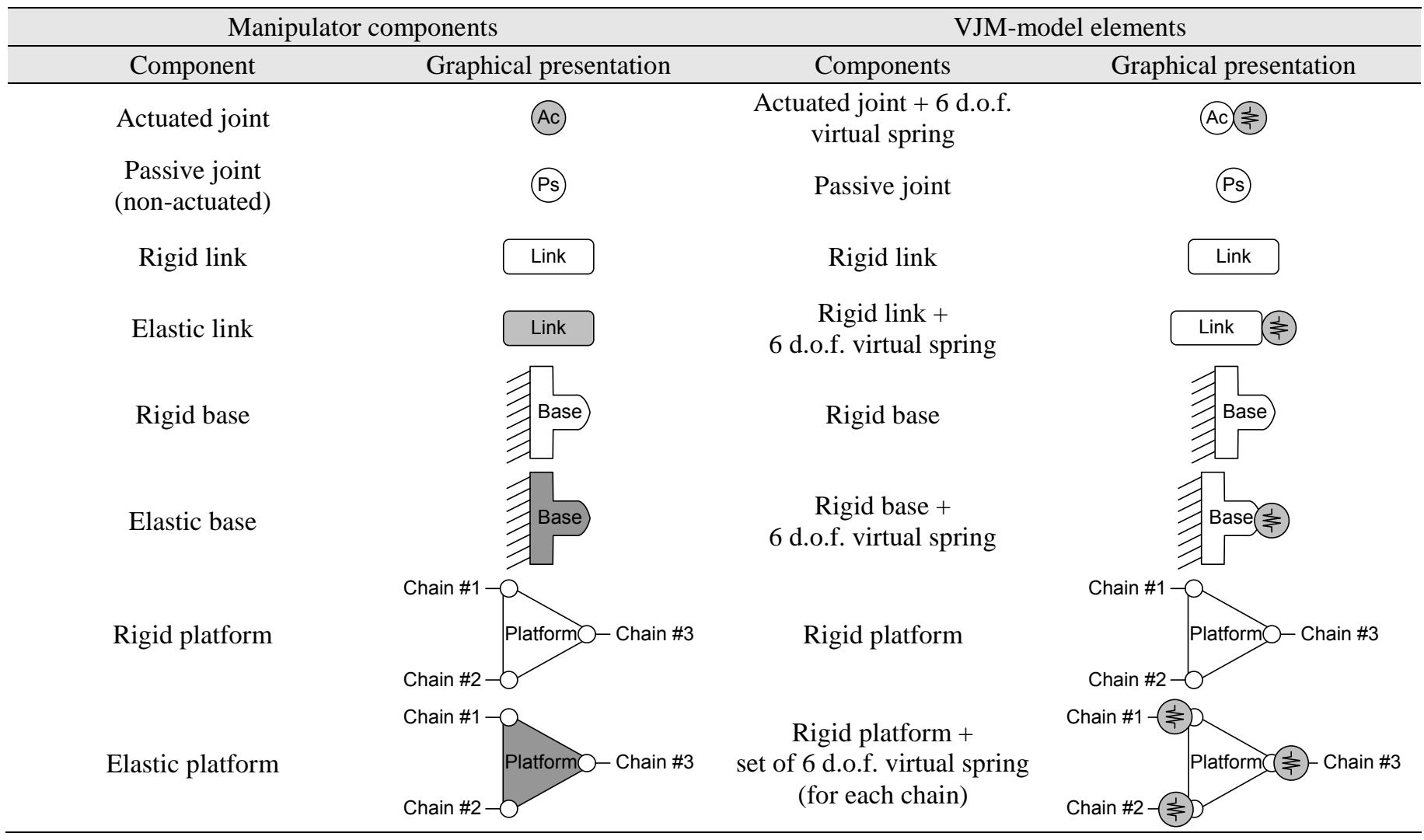

This technique originates from the work of Salisbury [39] who derived a closed-form expression for the Cartesian stiffness matrix of a serial manipulator assuming that the mechanical elasticity is concentrated in the actuated joints. The basic equations have been written as it follows

$$
\delta \mathbf{t}=\mathbf{J}_{\theta} \cdot \delta \boldsymbol{\theta} ; \quad \boldsymbol{\tau}=\mathbf{J}_{\theta}{ }^{\mathrm{T}} \cdot \mathbf{F} ; \quad \boldsymbol{\tau}=\mathbf{K}_{\theta} \cdot \delta \boldsymbol{\theta} ;
$$

where $\delta \mathbf{t}$ denotes the end-effector displacement in Cartesian space (both positional and orientational), $\mathbf{F}$ is the vector of the external loading applied to the end-effector (that includes both the force and the torque components), $\delta \boldsymbol{\theta}$ is the deflection in the virtual joint coordinates $\boldsymbol{\theta}$ caused by this loading, $\boldsymbol{\tau}$ is the vector of reactions in the elastic joints, $\mathbf{K}_{\theta}$ is the corresponding joint stiffness matrix, $\mathbf{J}_{\theta}$ is the Jacobian matrix computed with respect to the elastic joints. Here, the first equation is derived from the manipulator geometrical model, the second one describes the static equilibrium condition (assuming that the load is not essential), and the third equation presents the linear elasticity relation (Hooke's law).

After relevant transformations of (1), the desired linear relation between the external loading $\mathbf{F}$ and the end-effector displacement $\delta \mathbf{t}$ is presented as

$$
\delta \mathbf{t}=\left(\mathbf{J}_{\theta} \cdot \mathbf{K}_{\theta}^{-1} \cdot \mathbf{J}_{\theta}{ }^{\mathrm{T}}\right) \cdot \mathbf{F},
$$

which gives Cartesian stiffness matrix

$$
\mathbf{K}_{\mathrm{C}}=\mathbf{J}_{\theta}^{-\mathrm{T}} \cdot \mathbf{K}_{\theta} \cdot \mathbf{J}_{\theta}^{-1}
$$

In literature $[26,27,45]$, the latter may be also presented in a slightly different form

$$
\mathbf{K}_{\theta}=\mathbf{J}_{\theta} \cdot \mathbf{K}_{\mathrm{C}} \cdot \mathbf{J}_{\theta}{ }^{\mathrm{T}}
$$

which sometimes is referred to as Conservative Congruency Transformation (CCT), to emphasize that it describes mapping from the joint stiffness to Cartesian one, and vice versa.

In further works, similar equations were obtained for parallel manipulators assuming that they are not over-constrained and the elasticity is concentrated in the actuator joints while the passive joints are perfect [40]. Other contributions to this area include $[3,15,22,24,25,46-48]$, where the VJM method was partially extended. It is also worth mentioning the works where the VJM 
technique was applied to particular manipulators, in particular to the CaPAMan, Orthoglide and H4 robots, Stewart-Gough platforms, etc. [42,49-54].

VJM model parameters. In the first works, it was explicitly assumed that the main sources of elasticity are concentrated in actuated joints. Correspondingly, the links were assumed to be rigid and the VJM model included one-dimensional springs only. In other recent works, compliance of the links has been taken into account by introducing additional virtual joints describing their longitudinal elasticity [11] or stiffness properties in several directions [42]. Recent development in this area use 6-dimensional virtual joints to describe elasticity of each link [17].

At the beginning, the stiffness parameters of the virtual joints describing the link elasticity (and incorporated in the matrix $\mathbf{K}_{\theta}$ ) were evaluated rather roughly, using a simplified representation of the link shape by regular beams. Besides, it was assumed that all linear and angular deflections (compression/tension, bending, torsion) are decoupled and are presented by independent one-dimensional springs that produce a diagonal stiffness matrix of size $6 \times 6$ for each link. Afterwards, this elasticity model was enhanced by using complete $6 \times 6$ non-diagonal stiffness matrix of the cantilever beam [17,55]. This allowed taking into account all types of the translational/rotational compliance and relevant coupling between different deflections. Other enhancements include the link approximation by several beams, but it gives rather modest improvement in accuracy.

Further advance in this direction (applicable to the links of complicated shape) led to the FEA-based identification technique that involves virtual loading experiments in CAD environment and stiffness matrix estimation using dedicated numerical routines [34]. The latter essentially increased accuracy of the VJM-modeling while preserving its high computational efficiency. It is worth mentioning that usual high computational expenses of the FEA is not a critical issue here, because it is applied only once for each link (in contrast to the straightforward the FEA-modeling for the entire manipulator, which requires complete re-computing for each manipulator posture). As a result, this approach allowed the authors to integrate accuracy of the FEA-modeling into the VJM-modeling technique that provides high computational efficiency. General methodology of this hybrid approach is presented in Figure 2.

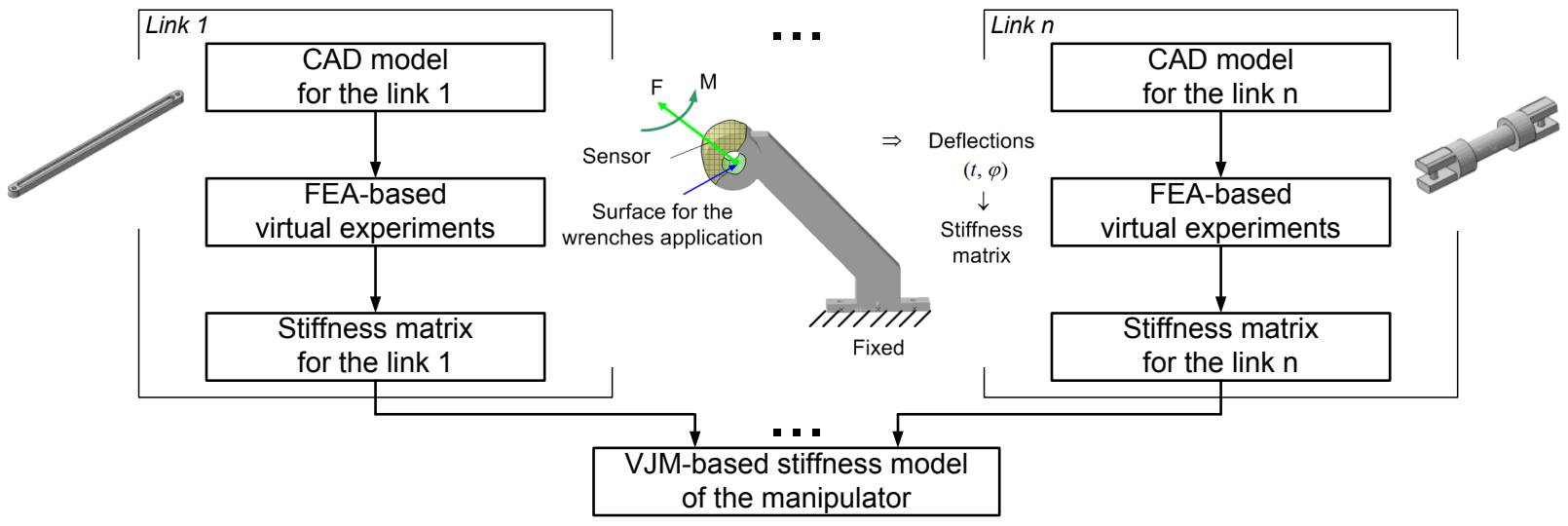

Figure 2 Integration of VJM modeling approach with FEA-based identification technique of the stiffness model parameters

Stiffness matrix for manipulators with passive joints. Another important issue is related to taking into account influence of the passive joints, which are widely used in parallel manipulators. In the simplest case, when the geometrical constraints imposed by the manipulator assembly are not redundant, the passive joint coordinates may be just eliminated from the kinematic model, allowing us direct application of the Salisbury formula. However, in the case of over-constrained or under-constrained manipulators, the elimination technique cannot be used directly.

For serial kinematic chains with passive joints, the problem has been solved for the general case [56]. In particular, it was proposed an algorithmic solution that extends the Salisbury formula and is able to produce the rank-deficient stiffness matrices describing "zero-resistance" of the end-effector to certain types of displacements, which do not require deflections in the virtual springs (due to presence of passive joints and/or kinematic singularity of the examined posture). The relevant technique involves inversion of dedicated square matrix of size $\left(n_{\theta}+6\right) \times\left(n_{\theta}+6\right)$, which is composed of the links stiffness matrices, and kinematic Jacobians of both virtual springs and passive joints (here $n_{\theta}$ is the number of passive joints). Then, the desired Cartesian stiffness matrix is obtained by simple extraction of an appropriate $6 \times 6$ sub-matrix from the computed inverse. Corresponding expression for stiffness matrix computation can be presented as

$$
\left[\begin{array}{cc}
\mathbf{K}_{\mathrm{C}} & * \\
* & *
\end{array}\right]=\left[\begin{array}{cc}
\mathbf{J}_{\theta} \mathbf{K}_{\theta}^{-1} \mathbf{J}_{\theta}^{\mathrm{T}} & \mathbf{J}_{\mathrm{q}} \\
\mathbf{J}_{\mathrm{q}}^{\mathrm{T}} & \mathbf{0}
\end{array}\right]^{-1}
$$


where $\mathbf{J}_{\theta}$ is Jacobian related to the virtual springs $\left(6 \times n_{\theta}\right), \mathbf{J}_{q}$ is Jacobian related to the passive joints $\left(6 \times n_{q}\right)$. The main advantage of this method is its computational simplicity, since the number of the virtual springs do not influence on the size of the matrix to be inverted. Besides, the method does not require manual elimination of the redundant spring corresponding to the passive joints, since this operation is inherently included in the numerical algorithm.

Because of its evident advantages, this approach found further development [4,57]. In these works, an analytical expression for the matrix $\mathbf{K}_{\mathrm{C}}$ has been derived from (5) using the blockwise inversion and presented as

$$
\mathbf{K}_{\mathrm{C}}=\mathbf{K}_{\mathrm{C}}^{0}-\mathbf{K}_{\mathrm{C}}^{0} \cdot \mathbf{J}_{\mathrm{q}} \cdot\left(\mathbf{J}_{\mathrm{q}}^{\mathrm{T}} \cdot \mathbf{K}_{\mathrm{C}}^{0} \cdot \mathbf{J}_{\mathrm{q}}\right)^{-1} \cdot \mathbf{J}_{\mathrm{q}}^{\mathrm{T}} \cdot \mathbf{K}_{\mathrm{C}}^{0}
$$

where the first term $\mathbf{K}_{\mathrm{C}}^{0}=\left(\mathbf{J}_{\theta} \cdot \mathbf{K}_{\theta}^{-1} \cdot \mathbf{J}_{\theta}^{\mathrm{T}}\right)^{-1}$ is the stiffness matrix of the corresponding serial chain without passive joints and the second term describes the impact of the passive joints. Another important contribution in this area is a recursive procedure that allows user to take into account the impact of passive joints sequentially (one by one or by specific groups). Relevant expression has the following form

$$
\mathbf{K}_{\mathrm{C}}^{i+1}=\mathbf{K}_{\mathrm{C}}^{i}-\mathbf{K}_{\mathrm{C}}^{i} \cdot \mathbf{J}_{\mathrm{q}}^{i} \cdot\left(\mathbf{J}_{\mathrm{q}}^{i \mathrm{~T}} \cdot \mathbf{K}_{\mathrm{C}}^{i} \cdot \mathbf{J}_{\mathrm{q}}^{i}\right)^{-1} \cdot \mathbf{J}_{\mathrm{q}}^{i \mathrm{~T}} \cdot \mathbf{K}_{\mathrm{C}}^{i} ; \quad i=1,2, \ldots
$$

where the matrices $\mathbf{J}_{q}^{i} \subset \mathbf{J}_{q}$ are extracted from the full Jacobian $\mathbf{J}_{q}=\left[\mathbf{J}_{q}^{1}, \mathbf{J}_{q}^{2}, \ldots\right]$ in arbitrary order (column-by-column, or by groups of columns).

However, for parallel manipulators with passive joints, solutions were obtained for less general cases. They include "pure" parallel architectures where the base and the end platform are connected by strictly serial kinematic chains. Here, the total stiffness matrix can be presented as the sum of partial matrices corresponding to separate chains (computed using the above described technique)

$$
\mathbf{K}_{c}=\sum_{i} \mathbf{K}_{\mathrm{C}}^{(i)} ; \quad\left[\begin{array}{cc}
\mathbf{K}_{\mathrm{C}}^{(i)} & * \\
* & *
\end{array}\right]=\left[\begin{array}{cc}
\mathbf{J}_{\theta i} \mathbf{K}_{\theta i}^{-1} \mathbf{J}_{\theta i}^{T} & \mathbf{J}_{\mathrm{q} i} \\
\mathbf{J}_{\mathrm{q} i}^{\mathrm{T}} & \mathbf{0}
\end{array}\right]^{-1}
$$

so the passive joints are taken into account easily. Besides, in this case the over-constraining of the mechanism does not create additional difficulties. For instance, for the over-constrained manipulator of Orthoglide family [58], each of the parallel chains yields the stiffness matrix of rank 4 while their aggregation gives the matrix of full rank 6 . But if there exists a cross-linking between the parallel chains (like in kinematic parallelograms, for instance), this method can not be applied directly. For this case, some interesting results are presented in $[6,41]$ where the geometrical constraints were treated in a general way but detailed computational techniques were not developed.

VJM modeling of parallel manipulators: problem of internal stresses. In spite of the fact that the VJM technique has been originally developed for serial manipulators, it can be efficiently applied to parallel robots. The basic idea here is to obtain first the stiffness models of each kinematic chain separately, and after, to integrate them in a united model corresponding to the parallel manipulator. This idea was partially implemented in $[3,48]$, where the manipulator structure was assumed to be strictly parallel (i.e. without internal loops) and the kinematic chains where assembled in the same end-point.

Under such assumptions, the stiffness matrix of the parallel manipulator can be computed via straightforward summation of the chain stiffness matrices

$$
\mathbf{K}_{\mathrm{C}}=\sum_{i=1}^{n} \mathbf{K}_{\mathrm{C}}^{(i)}
$$

where the index $i$ defines the kinematic chains and $n$ is the total number of chains. However, in more general (and practically important) cases where the kinematic chains are connected to different points of the end-platform, this formula cannot be applied directly. Besides, for parallel manipulators with parallelogram-based links, some essential modifications are required.

Other limitation of the existing results devoted to the parallel manipulators is related to the assumption that the assembling does not produce any internal stresses. But in practice, numerous errors are accumulated in serial chains [59] and they cause non-negligible internal forces in manipulator joints (even if the external force applied to the end-effector is equal to zero). The internal forces may essentially change the manipulator behavior (modify the stiffness matrix, change the end-effector location, etc.) and should be obviously taken into account in the stiffness model. However, existing works ignore this issue. Another research issue is associated with stiffness modeling of parallel manipulators under loading, which has not received proper attention yet. 


\subsection{Stiffness modeling under external and internal loadings}

Manipulator stiffness modeling in the loaded mode is a relatively new research area, which is worth to be considered separately. This subsection presents analysis of related works and defines some important research problems that will be in the focus of this work. Some of them are based on the analogy that can be established between robotics and structural mechanics (it concerns buckling phenomena, for instance).

Types of loadings. Manipulator loading may be of different nature. For the stiffness modeling, it is reasonable to distinguish two main types of loading, external and internal ones. The external loading is caused mainly by an interaction between the robot end-effector and the workpiece, which is processed or transported in the considered technological process [2,26]. Another type of external loading exists due to the gravity influence on the manipulator links, for many heavy manipulators employed in machining the link weight is not negligible [5,7]. Besides, to compensate in a certain degree the gravity influence, some manipulators include special mechanisms generating external forces/torques in the opposite direction. It is worth mentioning that the external loading generated by a technological process is always applied to the manipulator end-effector while others may be applied at intermediate points (at joints, for instance). Besides, the external loadings caused by gravity have obvious distributed nature.

In addition to the above mentioned forces/torques, internal loading in some joints may exists. For instance, to eliminate backlash, the joints may include preloaded springs, which generate the force or torque even in standard "mechanical zero" configuration [9]. Though the internal forces/torques do not influence on the global equilibrium equations, they may change the equilibrium configuration and have influence on the manipulator stiffness properties. For this reason, internal preloading is used sometimes to improve the manipulator stiffness, especially in the neighborhood of kinematic singularities. Another case where the internal loading exists by default, is related to over-constrained manipulators that are subject of the so-called antagonistic actuating $[8,60]$. Here, redundant actuators generate internal forces and torques that are equilibrated in the frame of close loops.

It is obvious that the both external and internal loadings influence on the manipulator equilibrium configuration and, consequently, may modify the stiffness properties. So, they must undoubtedly be taken into account while developing the stiffness model.

Stiffness matrix for the loaded manipulator. At present, in most of related works the stiffness is evaluated in a quasi-static configuration with no external or internal loading. There is a very limited number of publications that directly address the loaded mode case (or so-called case of "large deflections"), where in addition to the conventional "elastic stiffness" in the joints it is necessary to take into account the "geometrical stiffness" arising due to the change in the manipulator configuration under the load. Although the existence of this additional stiffness component for elastic structures has been known for a long time [61], the importance of this problem for robotic manipulators has been highlighted rather recently. The most essential results in this area were obtained in $[1,2,62]$ where there are presented both some theoretical issues and several case studies for serial and parallel manipulators. Several authors $[8,9,60]$ addressed the problem of stiffness analysis for the manipulators with internal preloading or antagonistic actuating, but in relevant equations some of the second order kinematic derivates were neglected. Using notation adopted in this work and summarizing existing results $[1,2,13]$, the manipulator stiffness matrix for the loaded can be expressed as follows

$$
\mathbf{K}_{\mathrm{C}}=\mathbf{J}_{\theta}^{-\mathrm{T}}\left(\mathbf{K}_{\theta}-\mathbf{K}_{\mathrm{F}}\right) \mathbf{J}_{\theta}^{-1}
$$

where $\mathbf{K}_{\mathrm{F}}$ is $n_{\theta} \times n_{\theta}$ stiffness matrix that is induced by external loading and is not presented in previous equation (3). This matrix depends on both the derivatives of the Jacobian $\mathbf{J}_{\theta}$ and the loading vector $\mathbf{F}$. Required details concerning computing of $\mathbf{K}_{\mathrm{F}}$ are given in [1].

In the frame of the same concept, the manipulator stiffness model for the loaded mode was proposed in [5,7], where numerous factors were taken into account (conventional external loading, gravity forces, antagonistic redundant actuation, etc.). The final results for the stiffness matrix is presented as

$$
\mathbf{K}_{\mathrm{C}}=\mathbf{J}_{\theta}^{-\mathrm{T}} \mathbf{K}_{\mathrm{u}} \mathbf{J}_{\theta}^{-1}
$$

where $\mathbf{K}_{u}$ is a solution of a non-linear matrix equation, which includes the joint stiffness and the external/internal loadings as parameters. However, this approach is rather hard from computational point of view. Besides, in this work the Jacobians and all their derivatives have been computed not in a "true" equilibrium configuration (it was unreasonably replaced by unloaded one). For this reason, since the equilibrium obviously depends on the loading magnitude, some essential issues were omitted. As a result, any nonlinear effects have been detected in the stiffness behavior of the examined manipulators.

Another significant result, which should be mentioned here, is related to the stiffness modeling of the parallel manipulators with the cross-linkage. Firstly this problem was considered in [5,7] where all coordinates where decomposed into two groups (dependent and independent ones). But no clear rule for such coordinate splitting has been proposed, besides the developed 
technique involved very non-trivial computations. Further, the cross-linkage was in the focus of $[6,41]$ where a rather compact expression for the stiffness matrix has been proposed

$$
\mathbf{K}_{\mathrm{C}}=\mathbf{J}_{\theta}^{-\mathrm{T}}\left(\mathbf{K}_{\theta}-\mathbf{K}_{\mathrm{F}}+\mathbf{K}_{\mathrm{I}}\right) \mathbf{J}_{\theta}^{-1}
$$

which, compared to (10), includes additional matrix $\mathbf{K}_{\mathrm{I}}$ that is induced by the geometrical constraints (cross-linkage). However, there are still a number of open questions concerning the coordinate splitting rule (i.e. dividing them into dependent and independent ones) and computing of the equilibrium configuration corresponding to the applied loading.

It should be noted that this problem of (computing the loaded equilibrium) has been omitted in most of the related works. At the same time, it is clear that the changes in the manipulator configuration directly influence the Jacobians (and their derivatives) as well as on the end-effector location. For this reason, computing the Jacobians and Hessians in a traditional way (i.e. for the unloaded configuration) may lead to excessively rough simplification of the stiffness model. In particular, some non-linear phenomena in manipulator stiffness behavior can be hardly detected, while they should obviously exist from the point of view general theory of elastic structures.

To our knowledge, the most extended results in this area has been presented in [10], where the authors proposed a non-linear stiffness model for parallel manipulators with passive joints which take into account deviations in the manipulator configuration caused by the external loading applied to the manipulator end-effector. In the frame of this conception, an iterative scheme that allows them to compute a static equilibrium configuration has been developed

$$
\left[\begin{array}{l}
\mathbf{F}_{i+1} \\
\mathbf{q}_{i+1} \\
\boldsymbol{\theta}_{i+1}
\end{array}\right]=\left[\begin{array}{ccc}
0 & \mathbf{J}_{\mathrm{q}} & \mathbf{J}_{\theta} \\
\mathbf{J}_{\mathrm{q}}^{T} & 0 & 0 \\
\mathbf{J}_{\theta}^{T} & 0 & -\mathbf{K}_{\theta}
\end{array}\right]^{-1}\left[\begin{array}{c}
\Delta \mathbf{t}+\mathbf{J}_{\mathrm{q}} \cdot \mathbf{q}_{i}+\mathbf{J}_{\theta} \cdot \boldsymbol{\theta}_{i} \\
0 \\
-\mathbf{K}_{\theta} \cdot \boldsymbol{\theta}_{0}
\end{array}\right]
$$

where the vector $\Delta \mathbf{t}$ defines the end-effector displacement caused by the external loading and $\boldsymbol{\theta}_{0}$ is preloading in virtual springs. Since, this procedure cannot distinguish a stable equilibrium configuration from an unstable one, there were proposed a matrix criteria that allows user to check stability via analyzing matrix properties

$$
\left[\begin{array}{c}
\mathbf{V}_{\theta}^{\circ} \\
\mathbf{V}_{q}^{\circ}
\end{array}\right]^{T} \cdot\left[\begin{array}{cc}
\mathbf{H}_{\theta \theta}^{F}-\mathbf{K}_{\theta} & \mathbf{H}_{q \theta}^{F} \\
\mathbf{H}_{\theta q}^{F} & \mathbf{H}_{q q}^{F}
\end{array}\right] \cdot\left[\begin{array}{l}
\mathbf{V}_{\theta}^{\circ} \\
\mathbf{V}_{\theta}^{\circ}
\end{array}\right]<0
$$

where $\mathbf{V}_{\theta}^{\circ}, \mathbf{V}_{q}^{\circ}$ are the sub-matrices corresponding to the zero singular values of aggregated Jacobian $\left[\mathbf{J}_{\theta}, \mathbf{J}_{q}\right]$ after applying to it SVD-factorization (see [10] for detail), $\mathbf{H}_{q q}^{F}, \mathbf{H}_{\theta \theta}^{F}, \mathbf{H}_{q \theta}^{F}, \mathbf{H}_{\theta q}^{F}$ are the Hessians of scalar function $\Psi=\mathbf{g}(\mathbf{q}, \boldsymbol{\theta})^{T} \cdot \mathbf{F}$ with respect to virtual and passive joint coordinates. In accordance with this criteria, the manipulator configuration is stable if (and only if) the matrix (14) is negative-definite. The stiffness matrix for the manipulator with passive joints under the end-point loading can be computed as

$$
\left[\begin{array}{c:c}
\mathbf{K}_{c} & * \\
\hdashline * & *
\end{array}\right]=\left[\begin{array}{cc}
\mathbf{J}_{\theta} \cdot \mathbf{k}_{\theta}^{F} \cdot \mathbf{J}_{\theta}^{T} & \mathbf{J}_{q}+\mathbf{J}_{\theta} \cdot \mathbf{k}_{\theta}^{F} \cdot \mathbf{H}_{\theta q}^{F} \\
\mathbf{J}_{q}^{T}+\mathbf{H}_{q \theta}^{F} \cdot \mathbf{k}_{\theta}^{F} \cdot \mathbf{J}_{\theta}^{T} & \mathbf{H}_{q q}^{F}+\mathbf{H}_{q \theta}^{F} \cdot \mathbf{k}_{\theta}^{F} \cdot \mathbf{H}_{\theta q}^{F}
\end{array}\right]^{-1}
$$

where $\mathbf{k}_{\theta}^{F}=\left(\mathbf{K}_{\theta}-\mathbf{H}_{\theta \theta}^{\mathbf{F}}\right)^{-1}$. Among the limitations of the proposed approach it should be mentioned that it is suitable for the manipulators under the loading applied to the end-effector only and with perfect serial chains. These limitations will be overcome in this paper.

Nonlinear-behavior of the manipulator under loading. In mechanics, it has been known since a long time that the elastic structures may suddenly change their configuration if the loading exceed some critical value. A classical example is the so-called Euler column that retains its straight shape until the loading. This effect (buckling) is well known in structural mechanics, however in robotics this aspect has never been studied before.

Non-linear behavior of force-deflection relation and possible buckling effects have been known since a long time. However in robotics, these questions did not attract much attention, mainly due to high rigidity of commercially available robots. But current trends in mechanical design of manipulators that are targeted at essential reduction of moving masses motivate relaxing this assumption. Hence, non-linear stiffness analysis is also important for the robotic manipulator. As it was mentioned before, existing stiffness analysis techniques for robots are strictly assumed that loading cannot change configurations of an examined manipulator or these changes are negligible. This simplification imposes crucial limitations for the stiffness analysis and, as a result, does not allow us to detect buckling and other non-linear phenomena known from general theory of elastic structures. 
Similar to the classical mechanics three types of buckling can appear in a robotic system: buckling of the link, contact buckling and geometrical buckling. First type of buckling is defined by the mechanical properties of the link and easily can be detected by FEA analysis or critical loading for it can be computed via approximated equations. Normally these loadings are unreachable in robots, while minimization of the link cross-sections can make these limits reachable. Thus it is reasonable to check critical loads for the buckling of elements on the design step. The second type of buckling is caused by the contact of the links with environment. It can be avoided on the machining process designing stage. The nature of the geometrical buckling is closed to the buckling of the elements, while several elements should be analysed together. In this case the critical force is defined by the stiffness of the links and junctions between them. Since stiffness of the junction may be lower than stiffness of links, or even in parallel manipulators can be negligible (for the passive joints), the critical force can be reduced in times comparing with the critical loading of the separate elements. So, nonlinear effects and buckling can appear in robots and they require additional analysis, however these questions have been omitted before.

In practice, it is impossible to detect non-linear effect without finding the loaded equilibrium, while this question was omitted in previous works. Besides, the loading may potentially lead to multiple equilibriums, to bifurcations of the equilibriums and to static instability of the manipulator configurations. These effects are essentialy dangerous for parallel manipulators wich impose numerious passive joints. Some aspects of multiple-equilibrium problem for robotic manipulators have been examined in the works $[63,64]$ who applied the Catastrophe theory for the stability analysis of the planar parallel manipulators with several flexural elements under external loading. However, they did not propose a general approach for stability analysis of the manipulator configurations. Therefore, it will be also in the focus of this research.

\section{Stiffness modeling for serial chain with internal and external loadings}

Typical examples of the examined kinematic chains can be found in the 3-PUU translational parallel kinematic machine [17], in the Delta parallel robot [65] or in the parallel manipulators of the Orthoglide family [58] and other manipulators [66]. It is worth mentioning that here a specific spatial arrangement of under-constrained chains yields the over-constrained mechanism that posses a high structural rigidity with respect to the external force. In particular, for Orthoglide, each kinematic chain prevents the platform from rotating around two orthogonal axes and any combination of two kinematic chains suppresses all possible rotations of the platform. Hence, the whole set of three kinematic chains produces a non-singular stiffness matrix while for each separate chain the stiffness matrix is singular. This motivated the development of dedicated stiffness analysis techniques that are presented below.

\subsection{Problem statement}

Let us consider a general serial kinematic chain, which consists of a fixed "Base", a number of flexible actuated joints "Ac", a serial chain of flexible "Links", a number of passive joints "Ps" and a moving "Platform" at the end of the chain (Figure 3). It is assumed that all links are separated by joints (actuated or passive, rotational or translational) and the joint type order is arbitrary. Besides, it is admitted that some links may be separated by actuated and passive joints simultaneously. Such architecture can be found in most of the parallel manipulators where several similar kinematic chains are connected to the same base and the platform in a different way (with the rotation of $90^{\circ}$ or $120^{\circ}$, for instance), in order to eliminate the redundancy caused by the passive joints. It is obvious that such kinematic chains are statically under-constrained and their stiffness analysis cannot be performed by the direct application of the standard methods.

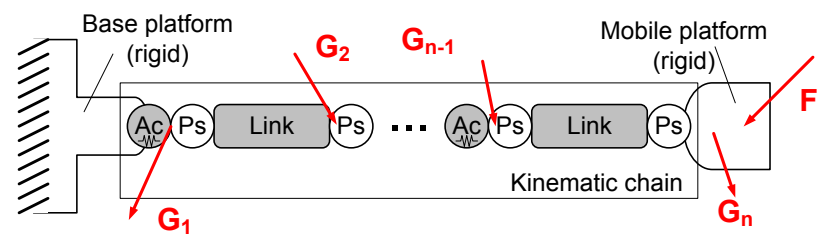

a) kinematic model

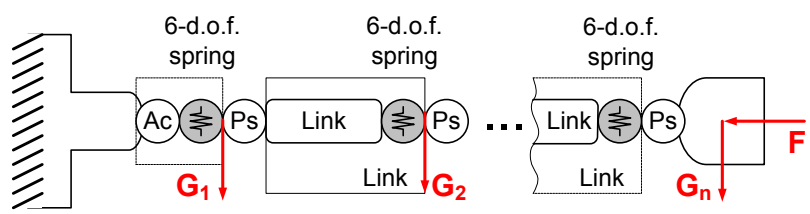

b) VJM model

Figure 3 General structure of kinematic chain with auxiliary loading and its VJM model

In order to evaluate the stiffness of the considered serial chain, let us apply a modification of the virtual joint method (VJM), which is based on the lump modelling approach [39,40]. According to this approach, the original rigid model should be extended by adding virtual joints (localized springs), which describe elastic deformations of the links. Besides, virtual springs are included in the actuating joints, to take into account the stiffness of the control loop. Under such assumptions, the kinematic chain can be described by the following serial structure:

(a) a rigid link between the manipulator base and the first actuating joint described by the constant homogenous transformation matrix $\mathbf{T}_{\text {Base }}$; 
(b) the 6-d.o.f. actuating joints defining three translational and three rotational actuator coordinates, which are described by the homogenous matrix function $\mathbf{T}_{3 \mathrm{D}}\left(\boldsymbol{\theta}_{\mathrm{a}}^{i}\right)$ where $\boldsymbol{\theta}_{\mathrm{a}}^{i}=\left(\theta_{\mathrm{x}}^{\mathrm{ai}}, \theta_{\mathrm{y}}^{\mathrm{a} i}, \theta_{\mathrm{z}}^{\mathrm{ai}}, \theta_{\varphi \mathrm{x}}^{\mathrm{ai}}, \theta_{\varphi \mathrm{y}}^{a i}, \theta_{\varphi \mathrm{z}}^{\mathrm{ai}}\right)$ are the virtual spring coordinates;

(c) the 6-d.o.f. passive joints defining three translational and three rotational passive joints coordinates, which are described by the homogenous matrix function $\mathbf{T}_{3 \mathrm{D}}\left(\mathbf{q}_{\mathrm{p}}^{i}\right)$ where $\mathbf{q}_{\mathrm{p}}^{i}=\left(q_{\mathrm{x}}^{i}, q_{\mathrm{y}}^{i}, q_{\mathrm{z}}^{i}, q_{\varphi \mathrm{x}}^{i}, q_{\varphi \mathrm{y}}^{i}, q_{\varphi \mathrm{z}}^{i}\right)$ are the passive joint coordinates;

(d) the rigid links, which are described by the constant homogenous transformation matrix $\mathbf{T}_{\text {Link }}^{i}$;

(e) a 6-d.o.f. virtual joint defining three translational and three rotational link-springs, which are described by the homogenous matrix function $\mathbf{T}_{3 \mathrm{D}}\left(\boldsymbol{\theta}_{\text {Link }}^{i}\right)$, where $\boldsymbol{\theta}_{\text {Link }}^{i}=\left(\theta_{\mathrm{x}}^{i}, \theta_{\mathrm{y}}^{i}, \theta_{\mathrm{z}}^{i}, \theta_{\varphi \mathrm{x}}^{i}, \theta_{\varphi \mathrm{y}}^{i}, \theta_{\varphi \mathrm{z}}^{i}\right),\left(\theta_{\mathrm{x}}^{i}, \theta_{\mathrm{y}}^{i}, \theta_{\mathrm{z}}^{i}\right)$ and $\left(\theta_{\varphi \mathrm{x}}^{i}, \theta_{\varphi \mathrm{y}}^{i}, \theta_{\varphi \mathrm{z}}^{i}\right)$ correspond to the elementary translations and rotations respectively;

(f) a rigid link from the last link to the end-effector, described by the homogenous matrix transformation $\mathbf{T}_{\text {Tоо1 }}$.

In the frame of these notations, the final expression defining the end-effector location subject to variations of all joint coordinates of a single kinematic chain may be written as the product of the following homogenous matrices

$$
\mathbf{T}=\mathbf{T}_{\text {Base }} \cdot \prod\left(\mathbf{T}_{3 \mathrm{D}}\left(\boldsymbol{\theta}_{\mathrm{a}}^{i}\right) \cdot \mathbf{T}_{3 \mathrm{D}}\left(\mathbf{q}_{\mathrm{p}}^{2 i-1}\right) \cdot \mathbf{T}_{\text {Link }}^{i} \cdot \mathbf{T}_{3 \mathrm{D}}\left(\mathbf{\theta}_{\text {Link }}^{i}\right) \cdot \mathbf{T}_{3 \mathrm{D}}\left(\mathbf{q}_{\mathrm{p}}^{2 i}\right)\right) \cdot \mathbf{T}_{\text {Tool }}
$$

where the components $\mathbf{T}_{\text {Base }}, \mathbf{T}_{3 \mathrm{D}}(\ldots), \mathbf{T}_{\text {Link }}^{i}, \mathbf{T}_{\text {Tоol }}$ may be factorized with respect to the terms including the joint variables, in order to simplify computing of the derivatives (Jacobian and Hessian).

This expression includes both traditional geometric variables (passive and active joint coordinates) and stiffness variables (virtual joint coordinates). The explicit position and orientation of the end-effector can by extracted from the matrix $\mathbf{T}$ in a standard way [19], so finally the kinematic model can be rewritten as the vector function

$$
\mathbf{t}=\mathbf{g}(\mathbf{q}, \boldsymbol{\theta})
$$

where the vector $\mathbf{t}=(\mathbf{p}, \boldsymbol{\varphi})^{\mathrm{T}}$ includes the position $\mathbf{p}=(x, y, z)^{\mathrm{T}}$ and orientation $\boldsymbol{\varphi}=\left(\varphi_{x}, \varphi_{\mathrm{y}}, \varphi_{z}\right)^{\mathrm{T}}$ of the end-platform, the vector $\mathbf{q}=\left(q_{1}, q_{2}, \ldots, q_{\mathrm{nq}}\right)^{T}$ contains all passive joint coordinates, the vector $\boldsymbol{\theta}=\left(\theta_{1}, \theta_{2}, \ldots, \theta_{\mathrm{n} \theta}\right)^{\mathrm{T}}$ collects all virtual joint coordinates, $n_{\mathrm{q}}$ is the number of the passive joins, $n_{\theta}$ is the number of the virtual joints.

Several examples of prismatic passive and actuated joints are presented in Figure 4a-c, some other types of joints have been illustrated in [67-70]. Such joints include internal springs, as such their statics is described by the following expression

$$
\tau_{\theta i}=K_{\theta i} \cdot\left(\theta_{i}-\theta_{0 i}\right)
$$

where $\tau_{\theta i}$ is the torque/force caused by the deviation of the joint coordinate $\theta_{i}$ from its unloaded ("zero") value $\theta_{0 i}$, and coefficient $K_{\theta i}$ defines the spring stiffness. For the purpose of generality, let us introduce similar "zero" values $\theta_{0 i}$ for the virtual springs that described flexibility of the links (obviously they are equal to zero for this subset of $\boldsymbol{\theta}$ ). This allows us to define vector $\boldsymbol{\theta}_{0}$ of the same size as $\boldsymbol{\theta}$ and to present the static equations corresponding to all variables (corresponding to perfect and preloaded passive joints, virtual springs of links and actuators) in general form

$$
\boldsymbol{\tau}_{\theta}=\mathbf{K}_{\theta} \cdot\left(\boldsymbol{\theta}-\boldsymbol{\theta}_{0}\right), \quad \boldsymbol{\tau}_{\mathrm{q}}=\mathbf{0}
$$

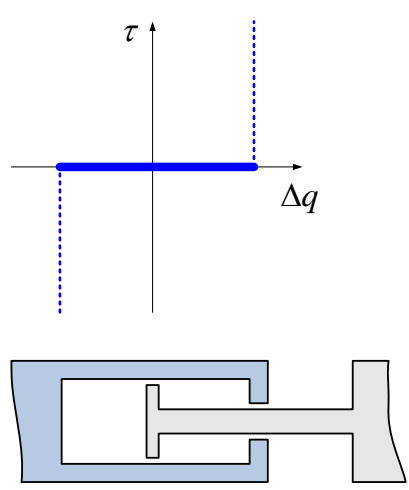

(a) Passive joint with hard end-constrains
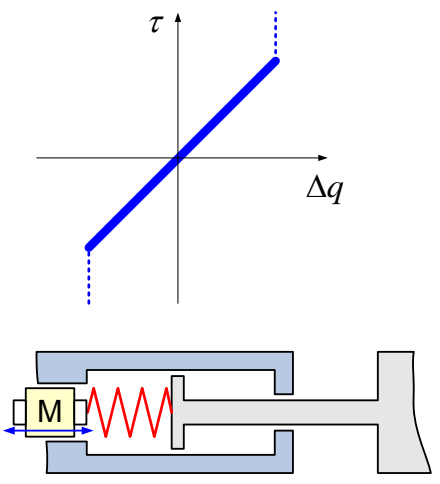

(b) Actuated joint with elastic transmission
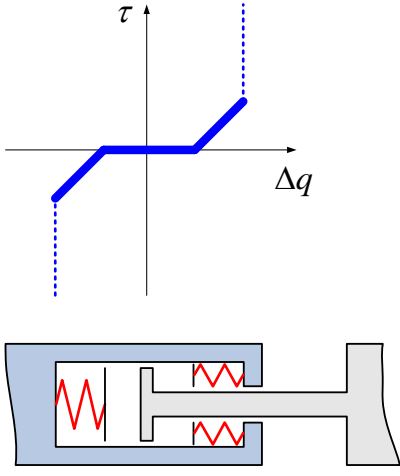

(a) Passive joint with soft end-constrains

Figure 4 Examples of prismatic passive and actuated joints 
Here $\boldsymbol{\tau}_{\theta}, \boldsymbol{\tau}_{q}$ are the generalized torque/force in joints corresponding to the variables $\boldsymbol{\theta}$ and $\mathbf{q}$; the matrix $\mathbf{K}_{\theta}$ collects stiffness coefficients of all springs of the kinematic chain. In the frame of this paper it is assumed that the internal loading (which may change the robot stiffness properties) may arise from two reasons: (i) due to additional elastic elements introduced by the designer in order to improve the manipulator properties; (ii) because of internal stresses in links/joints caused by over-constrained architecture of the considered manipulators. It is clear that here the total sum of all internal forces/torques is equal to zero (in contrast to the external loading studied in most of related works); nevertheless the internal loading influence on the manipulator stiffness matrix may be essential.

It is worth mentioning that in the case without internal preloading, the vector $\boldsymbol{\theta}$ describes only flexibility of manipulator links/actuators that are presented by virtual springs, while vector $\mathbf{q}$ collects entire set of passive joint coordinates. In contrast, here, the passive joint coordinates are divided into two subsets: (i) "perfect passive joints" included in q, and (ii) "preloaded passive joints" included in $\boldsymbol{\theta}$ together with traditional virtual springs. Besides, if a passive joint includes a nonlinear spring (see Figure 4c), the corresponding joint variable may be included either in $\boldsymbol{\theta}$ or $\mathbf{q}$, depending on the current configuration of the manipulator. However, for each configuration, this assignment is strictly unique.

It is assumed that the desired stiffness model of serial chain is defined by a non-linear relation

$$
\mathbf{F}=f(\Delta \mathbf{t}),
$$

where $f(\ldots)$ is a so-called "force-deflections" function that associates a deflection $\Delta \mathbf{t}$ with an external force $\mathbf{F}$ that causes deformations. It is worth mentioning that the function $f(\ldots)$ can be determined even for the singular configurations (or redundant kinematics) while the inverse statement is not generally true. Hence, enhanced stiffness analysis must include the computation of this function and the detailed analysis of its singularities that may provoke various nonlinear phenomena (such as buckling). In the unloaded case, this function is usually defined through the "stiffness matrix" $\mathbf{K}$, which describes the linear relation $\mathbf{F}=\mathbf{K}\left(\mathbf{q}_{0}, \boldsymbol{\theta}_{0}\right) \cdot \Delta \mathbf{t}$ between small six-dimensional translational/rotational displacements $\Delta \mathbf{t}$, and the external forces/torques $\mathbf{F}$ causing this transition. Here, it is assumed that $\Delta \mathbf{t}$ includes three positional components $(\Delta x, \Delta y, \Delta z)$ describing the displacement in Cartesian space and three angular components $\left(\Delta \varphi_{x}, \Delta \varphi_{y}, \Delta \varphi_{z}\right)$ that describe the end-platform rotation around the Cartesian axes, while the vectors $\mathbf{q}_{0}, \boldsymbol{\theta}_{0}$ correspond to the manipulator equilibrium configuration for which the loadings (both internal and external) are equal to zero. However, for the loaded mode, similar linear relation is defined in the neighborhood of another static equilibrium, which corresponds to a different manipulator configuration $(\mathbf{q}, \boldsymbol{\theta})$, that is modified by external forces/torques $\mathbf{F}$. Respectively, in this case, the stiffness model describes the relation between the increments of the force $\boldsymbol{\delta} \mathbf{F}$ and the position $\mathbf{\delta} \mathbf{t}$

$$
\boldsymbol{\delta} \mathbf{F}=\mathbf{K}^{F}(\mathbf{q}, \boldsymbol{\theta}) \cdot \boldsymbol{\delta} \mathbf{t}
$$

where $\mathbf{q}=\mathbf{q}_{\mathbf{0}}+\boldsymbol{\Delta} \mathbf{q}$ and $\boldsymbol{\theta}=\boldsymbol{\theta}_{\mathbf{0}}+\boldsymbol{\Delta} \boldsymbol{\theta}$ denote the new configuration of the manipulator, and $\boldsymbol{\Delta q}, \boldsymbol{\Delta \boldsymbol { \theta }}$ are the deviations in the coordinates $\mathbf{q}, \boldsymbol{\theta}$ respectively.

For stiffness modeling of serial kinematic chain with auxiliary loading let us assume that the serial chain has the additional external loadings applied to the internal node points (Figure 3). These loadings can be caused by gravity forces (generally they are distributed, but in practice they can be approximated by localized ones) and/or gravity compensators. These forces will be denoted as $\mathbf{G}_{j}$, where $j=1, \ldots, n$ is the node number in the serial chain starting from the fix base (here, $j=n$ corresponds to the end-point). It should be noted that for computational convenience, it is assumed that the end point loading consists of two components $\mathbf{G}_{n}$ and $\mathbf{F}$ of different nature.

It is evident that in general the auxiliary forces $\mathbf{G}_{i}$ depend on the manipulator configuration. So, let us assume that they are described by the functions

$$
\mathbf{G}_{j}=\mathbf{G}_{j}(\mathbf{q}, \boldsymbol{\theta}),
$$

In contrast, for the external force $\mathbf{F}$, it is assumed that there is no direct relation with the manipulator configuration.

For the serial chains with the auxiliary loadings it is also required to extend the geometrical model. In particular, in addition to the equation (17) defining the end-point location, it is necessary to introduce the additional functions

$$
\mathbf{t}_{j}=\mathbf{g}_{j}(\mathbf{q}, \boldsymbol{\theta}), \quad j=1, \ldots, n
$$

defining locations of the nodes. It is worth mentioning that for the serial chain, the position $\mathbf{t}_{j}$ depends on a sub-set of the joint coordinates (corresponding to the passive and virtual joints located between the base and the $j$-th node), but for the purpose of analytical simplicity let us use the whole set of the joint coordinates $(\mathbf{q}, \boldsymbol{\theta})$ as the arguments of the functions $\mathbf{g}_{i}(\ldots)$.

Using these assumptions, the problem of stiffness modeling of serial chains with auxiliary loadings can be split in the following sub-problems: (a) deriving the static equilibrium equations for the chain with auxiliary loadings; (b) computing 
full-scale "force-deflections relation" for the end-point and intermediate nodes; (c) linearization of the relevant force-deflection relations in the neighborhood of the equilibrium and computing corresponding stiffness matrix. Let us focus on these sub-problems.

\subsection{Static equilibrium equations for serial chain with auxiliary loadings}

To obtain a desired stiffness model, let us derive first the static equilibrium equations. In the frame of this work, the notion of static equilibrium of serial chain is referred to the configuration (defined by a set of the actuated, virtual and passive joint coordinates) that depends on external/auxiliary loadings, which ensure zero sums of forces/torques for each link separately. Let us apply the principle of the virtual work and assume that the kinematic chain under external loadings $\mathbf{F}$ and $\mathbf{G}_{=}\left[\mathbf{G}_{1} \ldots \mathbf{G}_{n}\right]$ has the configuration $(\mathbf{q}, \boldsymbol{\theta})$ and the locations of the end-point and the nodes are $\mathbf{t}=\mathbf{g}(\mathbf{q}, \boldsymbol{\theta})$ and $\mathbf{t}_{j}=\mathbf{g}_{j}(\mathbf{q}, \boldsymbol{\theta}), j=\overline{1, n}$ respectively.

According to the principle of virtual work, the work of external forces $\mathbf{G}, \mathbf{F}$ is equal to the work of internal forces $\boldsymbol{\tau}_{\theta}$ caused by displacement of the virtual springs $\delta \boldsymbol{\theta}$

$$
\sum_{j=1}^{n}\left(\mathbf{G}_{j}{ }^{\mathrm{T}} \cdot \delta \mathbf{t}_{j}\right)+\mathbf{F}^{\mathrm{T}} \cdot \delta \mathbf{t}=\boldsymbol{\tau}_{\theta}{ }^{\mathrm{T}} \cdot \delta \boldsymbol{\theta}
$$

where the virtual displacements $\delta \mathbf{t}_{j}$ can be computed from the linearized geometrical model derived from (23)

$$
\delta \mathbf{t}_{j}=\mathbf{J}_{\theta}^{(j)} \cdot \delta \boldsymbol{\theta}+\mathbf{J}_{\mathrm{q}}^{(j)} \cdot \delta \mathbf{q}, \quad j=1 . . n,
$$

which includes the Jacobian matrices

$$
\mathbf{J}_{\theta}^{(j)}=\frac{\partial}{\partial \boldsymbol{\theta}} \mathbf{g}_{j}(\mathbf{q}, \boldsymbol{\theta}) ; \quad \mathbf{J}_{\mathrm{q}}^{(j)}=\frac{\partial}{\partial \mathbf{q}} \mathbf{g}_{j}(\mathbf{q}, \boldsymbol{\theta})
$$

with respect to the virtual and passive joint coordinates respectively.

Substituting (25) to (24) we can obtain the equation

$$
\sum_{j=1}^{n}\left(\mathbf{G}_{j}{ }^{\mathrm{T}} \cdot \mathbf{J}_{\theta}^{(j)} \cdot \delta \boldsymbol{\theta}+\mathbf{G}_{j}{ }^{\mathrm{T}} \cdot \mathbf{J}_{\mathrm{q}}^{(j)} \cdot \delta \mathbf{q}\right)+\left(\mathbf{F}^{\mathrm{T}} \cdot \mathbf{J}_{\theta}^{(n)} \cdot \delta \boldsymbol{\theta}+\mathbf{F}^{\mathrm{T}} \cdot \mathbf{J}_{\mathrm{q}}^{(n)} \cdot \delta \mathbf{q}\right)=\boldsymbol{\tau}_{\theta}{ }^{\mathrm{T}} \cdot \delta \boldsymbol{\theta}
$$

which has to be satisfied for any variation of $\delta \boldsymbol{\theta}, \delta \mathbf{q}$. It means that the terms regrouping the variables $\delta \boldsymbol{\theta}, \delta \mathbf{q}$ have the coefficients equal to zero, hence the force-balance equations can be written as

$$
\boldsymbol{\tau}_{\theta}=\sum_{j=1}^{n} \mathbf{J}_{\theta}^{(j) \mathrm{T}} \cdot \mathbf{G}_{j}+\mathbf{J}_{\theta}^{(n) \mathrm{T}} \cdot \mathbf{F} ; \quad \mathbf{0}=\sum_{j=1}^{n} \mathbf{J}_{\mathrm{q}}^{(j) \mathrm{T}} \cdot \mathbf{G}_{j}+\mathbf{J}_{\mathrm{q}}^{(n) \mathrm{T}} \cdot \mathbf{F} .
$$

Also, these equations can be re-written in block-matrix form as

$$
\boldsymbol{\tau}_{\theta}=\mathbf{J}_{\theta}^{(\mathrm{G}) \mathrm{T}} \cdot \mathbf{G}+\mathbf{J}_{\theta}^{(\mathrm{F}) \mathrm{T}} \cdot \mathbf{F} ; \quad \mathbf{0}=\mathbf{J}_{\mathrm{q}}^{(\mathrm{G}) \mathrm{T}} \cdot \mathbf{G}+\mathbf{J}_{\mathrm{q}}^{(\mathrm{F}) \mathrm{T}} \cdot \mathbf{F}
$$

where

$$
\mathbf{J}_{\theta}^{(\mathrm{F})}=\mathbf{J}_{\theta}^{(n)} ; \quad \mathbf{J}_{\mathrm{q}}^{(F)}=\mathbf{J}_{\mathrm{q}}^{(n)} ; \quad \mathbf{J}_{\theta}^{(\mathrm{G})}=\left[\mathbf{J}_{\theta}^{(1) \mathrm{T}} \ldots \mathbf{J}_{\theta}^{(n) \mathrm{T}}\right]^{\mathrm{T}} ; \quad \mathbf{J}_{\mathrm{q}}^{(\mathrm{G})}=\left[\mathbf{J}_{\mathrm{q}}^{(1) \mathrm{T}} \ldots \mathbf{J}_{\mathrm{q}}^{(n) \mathrm{T}}\right]^{\mathrm{T}} ; \quad \mathbf{G}=\left[\mathbf{G}_{1}{ }^{\mathrm{T}} \ldots \mathbf{G}_{n}{ }_{n}{ }^{\mathrm{T}}\right]^{\mathrm{T}}
$$

Finally, taking into account the virtual spring reaction $\boldsymbol{\tau}_{\theta}=\mathbf{K}_{\theta} \cdot\left(\boldsymbol{\theta}-\boldsymbol{\theta}^{0}\right)$, where $\mathbf{K}_{\theta}=\operatorname{diag}\left(\mathbf{K}_{\theta_{1}}, \ldots, \mathbf{K}_{\theta_{\mathrm{n}}}\right)$, the desired static equilibrium equations can be presented as

$$
\begin{aligned}
& \mathbf{J}_{\theta}^{(\mathrm{G}) \mathrm{T}} \cdot \mathbf{G}+\mathbf{J}_{\theta}^{(\mathrm{F}) \mathrm{T}} \cdot \mathbf{F}=\mathbf{K}_{\theta} \cdot\left(\boldsymbol{\theta}-\boldsymbol{\theta}^{0}\right) \\
& \mathbf{J}_{\mathrm{q}}^{(\mathrm{G}) \mathrm{T}} \cdot \mathbf{G}+\mathbf{J}_{\mathrm{q}}^{(\mathrm{F}) \mathrm{T}} \cdot \mathbf{F}=\mathbf{0}
\end{aligned}
$$

It should be noted that compared to the case of end-effector loading only [10], here there are two additional terms $\mathbf{J}_{\theta}^{(\mathrm{G}) \mathrm{T}} \mathbf{G}$ and $\mathbf{J}_{\mathrm{q}}^{(\mathrm{G}) \mathrm{T}} \mathbf{G}$ that take into account the influence of the auxiliary loading $\mathbf{G}$. Further, these equations will be used for computing the static equilibrium configuration and corresponding Cartesian stiffness matrix. 


\subsection{Equilibrium configuration for serial chain with auxiliary loadings}

To obtain a relation between the external loading $\mathbf{F}$ and internal coordinates of the kinematic chain (q,, $\boldsymbol{\theta})$ corresponding to the static equilibrium, equations (31) should be solved either for the different given values of $\mathbf{F}$ or for the different given values of $\mathbf{t}$. Based on this data, the desired value of the end-point displacement can be computed straightforwardly, using geometric equation (17). In previous works, this issue was usually ignored and the linearization was performed in the neighborhood of the unloaded configuration assuming that the external load is small enough. It is obvious that the latter essentially limits relevant results and does not allow detecting non-linear effects such as buckling. From a mathematical point of view, the problem is reduced to solving of a system of the non-linear static equilibrium equations that may produce unique or non-unique, stable or unstable solutions.

For computational reasons, let us consider the dual problem that deals with determining the external force $\mathbf{F}$ and the manipulator equilibrium configuration $(\mathbf{q}, \boldsymbol{\theta})$ that corresponds to the end-effector location $\mathbf{t}$ taking into account internal preloading in the joints and auxiliary loading $\mathbf{G}(\mathbf{q}, \boldsymbol{\theta})$. Let us solve static equilibrium equations with respect to the manipulator configuration $(\mathbf{q}, \boldsymbol{\theta})$ and external loading $\mathbf{F}$ for given end-effector position $\mathbf{t}=\mathbf{g}(\mathbf{q}, \boldsymbol{\theta})$ and function of auxiliary-loadings $\mathbf{G}(\mathbf{q}, \boldsymbol{\theta})$

$$
\begin{array}{ll}
\mathbf{K}_{\theta} \cdot\left(\boldsymbol{\theta}-\boldsymbol{\theta}^{0}\right)=\mathbf{J}_{\theta}^{(\mathrm{G}) \mathrm{T}} \cdot \mathbf{G}+\mathbf{J}_{\theta}^{(\mathrm{F}) \mathrm{T}} \cdot \mathbf{F} ; & \mathbf{J}_{\mathrm{q}}^{(\mathrm{G}) \mathrm{T}} \cdot \mathbf{G}+\mathbf{J}_{\mathrm{q}}^{(\mathrm{F}) \mathrm{T}} \cdot \mathbf{F}=\mathbf{0} \\
\mathbf{t}=\mathbf{g}(\mathbf{q}, \boldsymbol{\theta}) ; & \mathbf{G}=\mathbf{G}(\mathbf{q}, \boldsymbol{\theta})
\end{array}
$$

Since this system of equations usually has no analytical solution, an iterative numerical technique can be applied. Similar to the previous Section, the kinematic equations may be linearized in the neighborhood of the current configuration $\left(\mathbf{q}_{i}, \boldsymbol{\theta}_{i}\right)$

$$
\mathbf{t}_{i+1}=\mathbf{g}\left(\mathbf{q}_{i}, \boldsymbol{\theta}_{i}\right)+\mathbf{J}_{\theta}^{(\mathrm{F})}\left(\mathbf{q}_{i}, \boldsymbol{\theta}_{i}\right) \cdot\left(\boldsymbol{\theta}_{i+1}-\boldsymbol{\theta}_{i}\right)+\mathbf{J}_{\mathrm{q}}^{(\mathrm{F})}\left(\mathbf{q}_{i}, \boldsymbol{\theta}_{i}\right) \cdot\left(\mathbf{q}_{i+1}-\mathbf{q}_{i}\right)
$$

where the subscript ' $i$ ' indicates the iteration number and the changes in Jacobians $\mathbf{J}_{\theta}^{(\mathrm{G})}, \mathbf{J}_{\theta}^{(\mathrm{F})}, \mathbf{J}_{\mathrm{q}}^{(\mathrm{G})}, \mathbf{J}_{\mathrm{q}}^{(\mathrm{F})}$ and the auxiliary loadings $\mathbf{G}(\mathbf{q}, \boldsymbol{\theta})$ are assumed to be negligible from iteration to iteration. Correspondingly, the static equilibrium equations in the neighborhood of $\left(\mathbf{q}_{i}, \boldsymbol{\theta}_{i}\right)$ may be rewritten as

$$
\begin{aligned}
& \mathbf{J}_{\theta}^{(\mathrm{G}) \mathrm{T}}\left(\mathbf{q}_{i}, \boldsymbol{\theta}_{i}\right) \cdot \mathbf{G}\left(\mathbf{q}_{i}, \boldsymbol{\theta}_{i}\right)+\mathbf{J}_{\theta}^{(\mathrm{F}) \mathrm{T}}\left(\mathbf{q}_{i}, \boldsymbol{\theta}_{i}\right) \cdot \mathbf{F}_{i+1}=\mathbf{K}_{\theta} \cdot\left(\boldsymbol{\theta}_{i+1}-\boldsymbol{\theta}^{0}\right) \\
& \mathbf{J}_{\mathrm{q}}^{(\mathrm{G}) \mathrm{T}}\left(\mathbf{q}_{i}, \boldsymbol{\theta}_{i}\right) \cdot \mathbf{G}\left(\mathbf{q}_{i}, \boldsymbol{\theta}_{i}\right)+\mathbf{J}_{\mathrm{q}}^{(\mathrm{F}) \mathrm{T}}\left(\mathbf{q}_{i}, \boldsymbol{\theta}_{i}\right) \cdot \mathbf{F}_{i+1}=\mathbf{0}
\end{aligned} .
$$

Thus, combining (33) and (34), the iterative algorithm for computing of the static equilibrium configuration for the given end-effector location can be presented as

$$
\left[\begin{array}{l}
\mathbf{F}_{i+1} \\
\mathbf{q}_{i+1} \\
\boldsymbol{\theta}_{i+1}
\end{array}\right]=\left[\begin{array}{ccc}
\mathbf{0} & \mathbf{J}_{\mathrm{q}}^{(\mathrm{F})}\left(\mathbf{q}_{i}, \boldsymbol{\theta}_{i}\right) & \mathbf{J}_{\theta}^{(\mathrm{F})}\left(\mathbf{q}_{i}, \boldsymbol{\theta}_{i}\right) \\
\mathbf{J}_{\mathrm{q}}^{(\mathrm{F}) \mathrm{T}}\left(\mathbf{q}_{i}, \boldsymbol{\theta}_{i}\right) & \mathbf{0} & \mathbf{0} \\
\mathbf{J}_{\theta}^{(\mathrm{F}) \mathrm{T}}\left(\mathbf{q}_{i}, \boldsymbol{\theta}_{i}\right) & \mathbf{0} & -\mathbf{K}_{\theta}
\end{array}\right]\left[\begin{array}{c}
\mathbf{t}_{i+1}-\mathbf{g}\left(\mathbf{q}_{i}, \boldsymbol{\theta}_{i}\right)+\mathbf{J}_{\theta}^{(\mathrm{F})}\left(\mathbf{q}_{i}, \boldsymbol{\theta}_{i}\right) \cdot \boldsymbol{\theta}_{i}+\mathbf{J}_{\mathrm{q}}^{(\mathrm{F})}\left(\mathbf{q}_{i}, \boldsymbol{\theta}_{i}\right) \cdot \mathbf{q}_{i} \\
-\mathbf{J}_{\mathrm{q}}^{(\mathrm{G}) \mathrm{T}}\left(\mathbf{q}_{i}, \boldsymbol{\theta}_{i}\right) \cdot \mathbf{G}_{i} \\
-\mathbf{J}_{\theta}^{(\mathrm{G}) \mathrm{T}}\left(\mathbf{q}_{i}, \boldsymbol{\theta}_{i}\right) \cdot \mathbf{G}_{i}-\mathbf{K}_{\theta} \cdot \boldsymbol{\theta}^{0}
\end{array}\right]
$$

where $\mathbf{G}_{i+1}=\mathbf{G}\left(\mathbf{q}_{i+1}, \boldsymbol{\theta}_{i+1}\right)$.

To reduce the size of a matrix to be inverted, the above system can be slightly simplified. In particular, applying the same approach as in [10] (but here based on analytical expression for $\left.\boldsymbol{\theta}=\mathbf{K}_{\theta}{ }^{-1}\left(\mathbf{J}_{\theta}^{(\mathrm{G}) \mathrm{T}} \cdot \mathbf{G}+\mathbf{J}_{\theta}^{(\mathrm{F}) \mathrm{T}} \cdot \mathbf{F}\right)+\boldsymbol{\theta}^{0}\right)$, the unknown variables can be separated in two groups $(\mathbf{F}, \mathbf{q})$ and $\boldsymbol{\theta}$. This yields the iterative scheme

$$
\begin{aligned}
{\left[\begin{array}{l}
\mathbf{F}_{i+1} \\
\mathbf{q}_{i+1}
\end{array}\right]=} & {\left[\begin{array}{cc}
\mathbf{J}_{\theta}^{(\mathrm{F})}\left(\mathbf{q}_{i}, \boldsymbol{\theta}_{i}\right) \cdot \mathbf{K}_{\theta}{ }^{-1} \cdot \mathbf{J}_{\theta}^{(\mathrm{F}) \mathrm{T}}\left(\mathbf{q}_{i}, \boldsymbol{\theta}_{i}\right) & \mathbf{J}_{\mathrm{q}}^{(\mathrm{F})}\left(\mathbf{q}_{i}, \boldsymbol{\theta}_{i}\right) \\
\mathbf{0}
\end{array}\right]^{-1} \times } \\
\mathbf{J}_{\mathrm{q}}^{(\mathrm{F}) \mathrm{T}}\left(\mathbf{q}_{i}, \boldsymbol{\theta}_{i}\right) & \left.-\mathbf{0}_{\mathrm{q}}\left(\mathbf{q}_{i}, \boldsymbol{\theta}_{i}\right) \cdot \mathbf{q}_{i}-\mathbf{J}_{\theta}^{(\mathrm{F})}\left(\mathbf{q}_{i}, \boldsymbol{\theta}_{i}\right) \cdot \mathbf{K}_{\theta}{ }^{-1} \cdot \mathbf{J}_{\theta}^{(\mathrm{G}) \mathrm{T}}\left(\mathbf{q}_{i}, \boldsymbol{\theta}_{i}\right) \cdot \mathbf{G}_{i}\right] \\
& \times\left[\begin{array}{c}
\left.\mathbf{t}_{i+1}-\mathbf{g}\left(\mathbf{q}_{i}, \boldsymbol{\theta}_{i}\right)+\mathbf{J}_{\theta}^{(\mathrm{F}) \mathrm{T}}\left(\mathbf{q}_{i}, \boldsymbol{\theta}_{i}\right) \cdot \boldsymbol{\theta}_{i}, \boldsymbol{\theta}_{i}\right) \cdot \mathbf{G}_{i} \\
\mathbf{\theta}_{i+1}^{(\mathrm{F})}=
\end{array}\right.
\end{aligned}
$$

The latter is more convenient computationally, since it requires the inversion of a lower dimension matrix $(n+6) \times(n+6)$ instead of $(n+m+6) \times(n+m+6)$, where $n, m$ are the sizes of the vectors $\mathbf{q}$ and $\boldsymbol{\theta}$ respectively. For instance, for the kinematic chains of the Orthoglide manipulator (see application example in Section 5), the expression (35) requires the inversion of $34 \times 34$ matrix, while iterative scheme (36) needs the inversion of $10 \times 10$ matrix only. It should be mentioned that $\mathbf{K}_{\theta}^{-1}$ is computed only once, outside of the iterative loop. 
Similar to the other iterative schemes, convergence of this algorithm highly depends on the starting point. However, due to the physical nature of the considered problem, it is possible to start iterations from the non-loaded configuration $\left(\mathbf{q}_{0}, \boldsymbol{\theta}_{0}\right)$. Besides, it is useful to modify the target point for each iteration in accordance with the expression $\mathbf{t}_{i}=\alpha_{i} \cdot \mathbf{t}+\left(1-\alpha_{i}\right) \cdot \mathbf{t}_{0}$ using scalar variable $\alpha_{i}$ that is monotonically increasing from 0 up to 1 . Another approach can be used for computing the force-deflection curve. Here, the starting point can be taken from previously computed loaded configuration corresponding to another value of deflection that is very close to the target one. For typical values of deformations, the proposed iterative procedure convergences in 3-5 iterations if the configuration is stable. In the simulation studies, the convergence has been evaluated by the weighted sum of residual norms corresponding to equations (36) and the algorithm stopped when this criterion achieved the prescribed value.

However, some computational difficulties may arise in the case of buckling or in the area of multiple equilibriums, where the convergence problem becomes rather critical and highly depends on an initial point. Here, the number of iterations increases significantly and the computational time becomes non-negligible. To overcome these difficulties, it is proposed to modify the developed iterative scheme and to repeat the computations several times, with slightly modified initial points that are obtained by adding small random noise to $\mathbf{q}_{0}, \boldsymbol{\theta}_{0}$. Another option is to add small disturbances to $\left(\boldsymbol{\theta}_{i}, \mathbf{q}_{i}\right)$ at each iteration. These techniques were used in the case studies presented in Section 5.

The proposed iterative scheme can also be slightly modified to solve the original problem, i.e. computing the equilibrium configuration corresponding to given external loading $\mathbf{F}$ (instead of given $\mathbf{t}$ ). In this case, expressions (35), (36) are used in the internal loop, while the desired algorithm is supplemented by an external loop, which provides iterative searching for $\mathbf{t}$ corresponding to the given $\mathbf{F}$

$$
\mathbf{t}_{i+1}=\mathbf{t}_{i}+\mathbf{K}_{i}^{-1} \cdot\left(\mathbf{F}-\mathbf{F}_{i}\right)
$$

where $\mathbf{t}_{i}, \mathbf{F}_{i}$ and $\mathbf{K}_{i}$ are the location, the loading and the stiffness matrix at the i-th iteration respectively. It is worth mentioning that the dual problem is meaningful only if the stiffness matrix $\mathbf{K}_{i}$ is non-singular. It is obvious that for a separate serial chain with passive joints the matrix $\mathbf{K}_{i}$ is always singular, while for an industrial serial manipulator it is usually non-singular (the same as for a parallel manipulator due to specific assembling of kinematic chains). On the other hand, the dual problem considered in this Section (i.e. computing $\mathbf{F}$ corresponding to $\mathbf{t}$ ) is always physically meaningful and has at least one solution.

Another problem related to computing equilibrium configuration that to be considered, is the stability of the manipulator configuration under the loading. This problem has been described in details in [10,71] and can be applied directly for the considered case since the matrix criterion (14) depends on the parameters that define the end-effector coordinates only; the impact of loadings applied to the intermediate points in this case is taken into account on the step of the equilibrium configuration computing .

Hence, the proposed algorithm allows us to compute static equilibrium configuration for the serial chains with passive joints and all types of loadings (internal preloading, external loadings applied to any point of the manipulator and loading from the technological process applied to the end-effector).

\subsection{Stiffness matrix for serial chain with auxiliary loadings}

The previous sub-section allows us to obtain the non-linear relation between elastic deflections $\Delta \mathbf{t}$ and external loading $\mathbf{F}$. Since common engineering practice operates with the stiffness matrix, let us linearize this relation in the neighborhood of the equilibrium. Following the virtual work technique, let us assume that the external force and the end-effector location are incremented by some small values $\delta \mathbf{F}, \delta \mathbf{t}$ in the neighborhood of current equilibrium configuration. Let us also assume that a new configuration also satisfies the equilibrium conditions. Hence, it is necessary to consider the two equilibriums corresponding to the manipulator state variables $(\mathbf{F}, \mathbf{q}, \boldsymbol{\theta}, \mathbf{t})$ and $(\mathbf{F}+\delta \mathbf{F}, \mathbf{q}+\delta \mathbf{q}, \boldsymbol{\theta}+\delta \boldsymbol{\theta}, \mathbf{t}+\delta \mathbf{t})$ simultaneously. The relevant static equilibrium equations may be written as

$$
\begin{aligned}
& \mathbf{t}=\mathbf{g}(\mathbf{q}, \boldsymbol{\theta}) \\
& \mathbf{K}_{\theta} \cdot\left(\boldsymbol{\theta}-\boldsymbol{\theta}^{0}\right)=\mathbf{J}_{\theta}^{(\mathrm{G}) \mathrm{T}} \cdot \mathbf{G}+\mathbf{J}_{\theta}^{(\mathrm{F}) \mathrm{T}} \cdot \mathbf{F} \\
& \mathbf{J}_{\mathrm{q}}^{(\mathrm{G}) \mathrm{T}} \cdot \mathbf{G}+\mathbf{J}_{q}^{(\mathrm{F}) \mathrm{T}} \cdot \mathbf{F}=\mathbf{0}
\end{aligned}
$$




$$
\begin{aligned}
& \mathbf{t}+\delta \mathbf{t}=\mathbf{g}(\mathbf{q}+\delta \mathbf{q}, \boldsymbol{\theta}+\delta \boldsymbol{\theta}) \\
& \mathbf{K}_{\theta} \cdot\left(\boldsymbol{\theta}+\delta \boldsymbol{\theta}-\boldsymbol{\theta}^{0}\right)=\left(\mathbf{J}_{\theta}^{(\mathrm{G})}+\delta \mathbf{J}_{\theta}^{(\mathrm{G})}\right)^{\mathrm{T}} \cdot(\mathbf{G}+\delta \mathbf{G})+\left(\mathbf{J}_{\theta}^{(\mathrm{F})}+\delta \mathbf{J}_{\theta}^{(\mathrm{F})}\right)^{\mathrm{T}} \cdot(\mathbf{F}+\delta \mathbf{F}) \\
& \left(\mathbf{J}_{\mathrm{q}}^{(\mathrm{G})}+\delta \mathbf{J}_{\mathrm{q}}^{(\mathrm{G})}\right)^{\mathrm{T}} \cdot(\mathbf{G}+\delta \mathbf{G})+\left(\mathbf{J}_{\mathrm{q}}^{(\mathrm{F})}+\delta \mathbf{J}_{\mathrm{q}}^{(\mathrm{F})}\right)^{\mathrm{T}} \cdot(\mathbf{F}+\delta \mathbf{F})=\mathbf{0}
\end{aligned}
$$

where the variables $\mathbf{t}, \mathbf{F}, \mathbf{G}, \mathbf{K}_{\theta}, \mathbf{q}, \boldsymbol{\theta}, \boldsymbol{\theta}^{0}$ are assumed to be known.

After linearization of the function $\mathbf{g}(\mathbf{q}, \boldsymbol{\theta})$ in the neighborhood of loaded equilibrium, the system (38), (39) is reduced to three equations

$$
\begin{aligned}
& \delta \mathbf{t}=\mathbf{J}_{\theta}^{(\mathrm{F})} \cdot \delta \boldsymbol{\theta}+\mathbf{J}_{\mathrm{q}}^{(\mathrm{F})} \cdot \delta \mathbf{q} \\
& \mathbf{K}_{\theta} \cdot \delta \boldsymbol{\theta}=\delta \mathbf{J}_{\theta}^{(\mathrm{G})} \cdot \mathbf{G}+\mathbf{J}_{\theta}^{(\mathrm{G})} \cdot \delta \mathbf{G}+\delta \mathbf{J}_{\theta}^{(\mathrm{F})} \cdot \mathbf{F}+\mathbf{J}_{\theta}^{(\mathrm{F})} \cdot \delta \mathbf{F} \\
& \delta \mathbf{J}_{\mathrm{q}}^{(\mathrm{G})} \cdot \mathbf{G}+\mathbf{J}_{\mathrm{q}}^{(\mathrm{G})} \cdot \delta \mathbf{G}+\delta \mathbf{J}_{\mathrm{q}}^{(\mathrm{F})} \cdot \mathbf{F}+\mathbf{J}_{\mathrm{q}}^{(\mathrm{F})} \cdot \delta \mathbf{F}=\mathbf{0}
\end{aligned}
$$

which define the desired linear relations between $\delta \mathbf{t}$ and $\delta \mathbf{F}, \delta \mathbf{q}, \delta \boldsymbol{\theta}$ that are expressed by the stiffness matrices $\mathbf{K}_{\mathrm{C}}, \mathbf{K}_{\mathrm{Cq}}$, $\mathbf{K}_{\mathrm{c} \theta}$. In this system, small variations of Jacobians may be expressed via the second order derivatives

$$
\begin{aligned}
\delta \mathbf{J}_{\mathrm{q}}^{(\mathrm{F})}=\mathbf{H}_{\mathrm{q} \theta}^{(\mathrm{F})} \cdot \delta \boldsymbol{\theta}+\mathbf{H}_{\mathrm{qq}}^{(\mathrm{F})} \cdot \delta \mathbf{q} ; & \delta \mathbf{J}_{\theta}^{(\mathrm{F})}=\mathbf{H}_{\theta \theta}^{(\mathrm{F})} \cdot \delta \boldsymbol{\theta}+\mathbf{H}_{\theta \mathrm{q}}^{(\mathrm{F})} \cdot \delta \mathbf{q} ; \\
\delta \mathbf{J}_{\mathrm{q}}^{(\mathrm{G})}=\mathbf{H}_{\mathrm{q} \theta}^{(\mathrm{G})} \cdot \delta \boldsymbol{\theta}+\mathbf{H}_{\mathrm{qq}}^{(\mathrm{G})} \cdot \delta \mathbf{q} ; & \delta \mathbf{J}_{\theta}^{(\mathrm{G})}=\mathbf{H}_{\theta \theta}^{(\mathrm{G})} \cdot \delta \boldsymbol{\theta}+\mathbf{H}_{\theta \mathrm{q}}^{(\mathrm{G})} \cdot \delta \mathbf{q} ;
\end{aligned}
$$

where

$$
\begin{array}{ll}
\mathbf{H}_{\theta \theta}^{(\mathrm{G})}=\sum_{j=1}^{n} \frac{\partial^{2}}{\partial \boldsymbol{\theta}^{2}}\left(\mathbf{g}_{j}{ }^{T}(\mathbf{q}, \boldsymbol{\theta}) \cdot \mathbf{G}_{j}\right) ; & \mathbf{H}_{\mathrm{qq}}^{(\mathrm{G})}=\sum_{j=1}^{n} \frac{\partial^{2}}{\partial \mathbf{q}^{2}}\left(\mathbf{g}_{j}{ }^{T}(\mathbf{q}, \boldsymbol{\theta}) \cdot \mathbf{G}_{j}\right) ; \\
\mathbf{H}_{\theta \mathrm{q}}^{(\mathrm{G})}=\sum_{j=1}^{n} \frac{\partial^{2}}{\partial \boldsymbol{\theta} \partial \mathbf{q}}\left(\mathbf{g}_{j}{ }^{T}(\mathbf{q}, \boldsymbol{\theta}) \cdot \mathbf{G}_{j}\right) ; & \mathbf{H}_{\mathrm{q} \theta}^{(\mathrm{G})}=\sum_{j=1}^{n} \frac{\partial^{2}}{\partial \mathbf{q} \partial \boldsymbol{\theta}}\left(\mathbf{g}_{j}^{T}(\mathbf{q}, \boldsymbol{\theta}) \cdot \mathbf{G}_{j}\right) ; \\
\mathbf{H}_{\theta \theta}^{(\mathrm{F})}=\frac{\partial^{2}}{\partial \boldsymbol{\theta}^{2}}\left(\mathbf{g}^{T}(\mathbf{q}, \boldsymbol{\theta}) \cdot \mathbf{F}\right) ; & \mathbf{H}_{\mathrm{qq}}^{(\mathrm{F})}=\frac{\partial^{2}}{\partial \mathbf{q}^{2}}\left(\mathbf{g}^{T}(\mathbf{q}, \boldsymbol{\theta}) \cdot \mathbf{F}\right) ; \\
\mathbf{H}_{\theta \mathrm{Fq}}^{(\mathrm{F})}=\frac{\partial^{2}}{\partial \boldsymbol{\theta} \partial \mathbf{q}}\left(\mathbf{g}^{T}(\mathbf{q}, \boldsymbol{\theta}) \cdot \mathbf{F}\right) ; & \mathbf{H}_{\mathrm{q} \theta}^{(\mathrm{F})}=\frac{\partial^{2}}{\partial \mathbf{q} \partial \boldsymbol{\theta}}\left(\mathbf{g}^{T}(\mathbf{q}, \boldsymbol{\theta}) \cdot \mathbf{F}\right) ;
\end{array}
$$

Also, the auxiliary loading $\mathbf{G}$ may be computed via the first order derivatives as

$$
\delta \mathbf{G}=\frac{\partial \mathbf{G}}{\partial \boldsymbol{\theta}} \cdot \delta \boldsymbol{\theta}+\frac{\partial \mathbf{G}}{\partial \mathbf{q}} \cdot \delta \mathbf{q}
$$

Furthermore, let us introduce the additional notation

$$
\begin{array}{lll}
\mathbf{H}_{\theta \theta}=\mathbf{H}_{\theta \theta}^{(\mathrm{F})}+\mathbf{H}_{\theta \theta}^{(\mathrm{G})}+\mathbf{J}_{\theta}^{(\mathrm{G}) \mathrm{T}} \cdot \frac{\partial}{\partial \boldsymbol{\theta}} \mathbf{G} ; & \mathbf{H}_{\theta q}=\mathbf{H}_{\theta \mathrm{q}}^{(\mathrm{G})}+\mathbf{H}_{\theta \mathrm{q}}^{(\mathrm{F})}+\mathbf{J}_{\theta}^{(\mathrm{G}) \mathrm{T}} \cdot \frac{\partial}{\partial \mathbf{q}} \mathbf{G} ; \\
\mathbf{H}_{\mathrm{q} \theta}=\mathbf{H}_{\mathrm{q} \theta}^{(\mathrm{G})}+\mathbf{H}_{\mathrm{q} \theta}^{(\mathrm{F})}+\mathbf{J}_{\mathrm{q}}^{(\mathrm{G}) \mathrm{T}} \cdot \frac{\partial}{\partial \boldsymbol{\theta}} \mathbf{G} ; & \mathbf{H}_{\mathrm{qq}}=\mathbf{H}_{\mathrm{qq}}^{(\mathrm{G})}+\mathbf{H}_{\mathrm{qq}}^{(\mathrm{F})}+\mathbf{J}_{\mathrm{q}}^{(\mathrm{G}) \mathrm{T}} \cdot \frac{\partial}{\partial \mathbf{q}} \mathbf{G}
\end{array}
$$

which allows us to present the system (40) in the form

$$
\left[\begin{array}{c}
\delta \mathbf{t} \\
\mathbf{0} \\
\mathbf{0}
\end{array}\right]=\left[\begin{array}{ccc}
\mathbf{0} & \mathbf{J}_{\mathrm{q}}^{(\mathrm{F})} & \mathbf{J}_{\theta}^{(\mathrm{F})} \\
\mathbf{J}_{\mathrm{q}}^{(\mathrm{F}) \mathrm{T}} & \mathbf{H}_{\mathrm{qq}} & \mathbf{H}_{\mathrm{q} \theta} \\
\mathbf{J}_{\theta}^{(\mathrm{F}) \mathrm{T}} & \mathbf{H}_{\theta q} & -\mathbf{K}_{\theta}+\mathbf{H}_{\theta \theta}
\end{array}\right] \cdot\left[\begin{array}{l}
\delta \mathbf{F} \\
\delta \mathbf{q} \\
\delta \boldsymbol{\theta}
\end{array}\right]
$$

The latter gives a straightforward numerical technique for computing the desired stiffness matrix: direct inversion of the matrix in the left-hand side of (45) and extracting from it the upper-left sub-matrix of size $6 \times 6$. Similarly, the matrices defining linear relations between the end-effector increment $\delta \mathbf{t}$ and the increments of the joint variables $\delta \boldsymbol{\theta}, \delta \mathbf{q}$ can be computed, i.e.:

$$
\delta \mathbf{F}=\mathbf{K}_{\mathrm{C}} \cdot \delta \mathbf{t} ; \quad \delta \boldsymbol{\theta}=\mathbf{K}_{\mathrm{C} \theta} \cdot \delta \mathbf{t} ; \quad \delta \mathbf{q}=\mathbf{K}_{\mathrm{Cq}} \cdot \delta \mathbf{t}
$$

where 


$$
\left[\begin{array}{c:c:c}
\mathbf{K}_{\mathrm{C}} & * & * \\
\hdashline \mathbf{K}_{\mathrm{Cq}} & * & * \\
\hdashline \mathbf{K}_{\mathrm{C} \theta} & * & *
\end{array}\right]=\left[\begin{array}{ccc}
\mathbf{0} & \mathbf{J}_{\mathrm{q}}^{(\mathrm{F})} & \mathbf{J}_{\theta}^{(\mathrm{F})} \\
\mathbf{J}_{\mathrm{q}}^{(\mathrm{F}) \mathrm{T}} & \mathbf{H}_{\mathrm{qq}} & \mathbf{H}_{\mathrm{q} \theta} \\
\mathbf{J}_{\theta}^{(\mathrm{F}) \mathrm{T}} & \mathbf{H}_{\theta \mathrm{q}} & -\mathbf{K}_{\theta}+\mathbf{H}_{\theta \theta}
\end{array}\right]^{-1}
$$

It is worth mentioning that the internal preloading (expressed by the variable $\boldsymbol{\theta}_{0}$ ) is not included in the latter expression in the explicit way, but it directly influences the variables $(\mathbf{q}, \boldsymbol{\theta})$ describing the equilibrium configuration and corresponding Jacobians and Hessians, which are elements of (47). Besides, in contrast to previous works, here it is possible to obtain supplementary matrices $\mathbf{K}_{\mathrm{C} \theta}, \mathbf{K}_{\mathrm{Cq}}$ that give additional measures of the manipulator stiffness which evaluate sensitivity of the joint coordinates $(\mathbf{q}, \boldsymbol{\theta})$ with respect to the external loading.

In the case when the matrix inverse (47) is computationally hard, the variable $\delta \boldsymbol{\theta}$ can be eliminated analytically, using corresponding static equation: $\delta \boldsymbol{\theta}=\mathbf{k}_{\theta}^{\mathrm{F}} \cdot \mathbf{J}_{\theta}^{(\mathrm{F}) \mathrm{T}} \cdot \delta \mathbf{F}+\mathbf{k}_{\theta}^{\mathrm{F}} \cdot \mathbf{H}_{\theta \mathrm{q}}^{\mathrm{F}} \cdot \delta \mathbf{q}$, where $\mathbf{k}_{\theta}^{\mathrm{F}}$ denotes the modified joint compliance matrix $\mathbf{k}_{\theta}^{\mathrm{F}}=\left(\mathbf{K}_{\theta}-\mathbf{H}_{\theta \theta}^{\mathrm{F}}\right)^{-1}$. This leads to a reduced system of matrix equations with unknowns $\delta \mathbf{F}$ and $\delta \mathbf{q}$

$$
\left[\begin{array}{cc}
\mathbf{J}_{\theta}^{(\mathrm{F})} \cdot \mathbf{k}_{\theta}^{\mathrm{F}} \cdot \mathbf{J}_{\theta}^{(\mathrm{F}) \mathrm{T}} & \mathbf{J}_{\mathrm{q}}^{(\mathrm{F})}+\mathbf{J}_{\theta}^{(\mathrm{F})} \cdot \mathbf{k}_{\theta}^{\mathrm{F}} \cdot \mathbf{H}_{\theta \mathrm{q}} \\
\mathbf{J}_{\mathrm{q}}^{(\mathrm{F}) \mathrm{T}}+\mathbf{H}_{\mathrm{q} \theta} \cdot \mathbf{k}_{\theta}^{\mathrm{F}} \cdot \mathbf{J}_{\theta}^{(\mathrm{F}) \mathrm{T}} & \mathbf{H}_{\mathrm{qq}}+\mathbf{H}_{\mathrm{q} \theta} \cdot \mathbf{k}_{\theta}^{\mathrm{F}} \cdot \mathbf{H}_{\theta \mathrm{q}}
\end{array}\right] \cdot\left[\begin{array}{c}
\delta \mathbf{F} \\
\delta \mathbf{q}
\end{array}\right]=\left[\begin{array}{c}
\delta \mathbf{t} \\
\mathbf{0}
\end{array}\right]
$$

that may be treated in the similar way, i.e. the desired stiffness matrix is also obtained by direct inversion of the matrix in the left-hand side of (48) and extracting from it the upper-left sub-matrix of size $6 \times 6$ :

$$
\left[\begin{array}{c:c}
\mathbf{K}_{\mathrm{C}} & * \\
\hdashline \mathbf{K}_{\mathrm{Cq}} & *
\end{array}\right]=\left[\begin{array}{cc}
\mathbf{J}_{\theta}^{(\mathrm{F})} \cdot \mathbf{k}_{\theta}^{\mathrm{F}} \cdot \mathbf{J}_{\theta}^{(\mathrm{F}) \mathrm{T}} & \mathbf{J}_{\mathrm{q}}^{(\mathrm{F})}+\mathbf{J}_{\theta}^{(\mathrm{F})} \cdot \mathbf{k}_{\theta}^{\mathrm{F}} \cdot \mathbf{H}_{\theta \mathrm{q}} \\
\mathbf{J}_{\mathrm{q}}^{(\mathrm{F}) \mathrm{T}}+\mathbf{H}_{\mathrm{q} \theta} \cdot \mathbf{k}_{\theta}^{\mathrm{F}} \cdot \mathbf{J}_{\theta}^{(\mathrm{F}) \mathrm{T}} & \mathbf{H}_{\mathrm{qq}}+\mathbf{H}_{\mathrm{q} \theta} \cdot \mathbf{k}_{\theta}^{\mathrm{F}} \cdot \mathbf{H}_{\theta \mathrm{q}}
\end{array}\right]^{-1}
$$

Similar to previous subsection, this approach allows us to reduce the dimension of the inverted matrix from $(n+m+6) \times(n+m+6)$ to $(n+6) \times(n+6)$, that in the case of Orthoglide corresponds to $34 \times 34$ and $10 \times 10$ respectively.

It is worth mentioning that the structure of the latter matrix is similar to the one obtained for the unloaded manipulator and for the manipulator under end-point loading only [10] and differs by Hessians that take into account the influence of the external load. It should also be noted that, because of the presence of the passive joints, the stiffness matrix of a separate serial kinematic chain is always singular, but aggregation of all the chains for a parallel manipulator produces a non-singular stiffness matrix.

Further simplification of (49) can be obtained by applying the block matrix inversion technique of Frobenius [72] that yields the following expressions

$$
\mathbf{K}_{\mathrm{C}}=\mathbf{K}_{C}^{0(F)}-\mathbf{K}_{C}^{0(F)} \cdot\left(\mathbf{J}_{\mathrm{q}}+\mathbf{J}_{\theta} \cdot \mathbf{k}_{\theta}^{\mathrm{F}} \cdot \mathbf{H}_{\theta \mathrm{q}}^{\mathrm{F}}\right) \cdot \mathbf{K}_{\mathrm{Cq}}
$$

where the first term $\mathbf{K}_{C}^{0(F)}=\left(\mathbf{J}_{\theta} \cdot \mathbf{k}_{\theta}^{\mathrm{F}} \cdot \mathbf{J}_{\theta}^{\mathrm{T}}\right)^{-1}$ exactly corresponds to the classical formula defining stiffness of the kinematic chain without passive joints in the loaded mode $[1,2]$ and

$$
\mathbf{K}_{\mathrm{Cq}}=-\left(\mathbf{H}_{\mathrm{qq}}^{\mathrm{F}}+\mathbf{H}_{\mathrm{q} \theta}^{\mathrm{F}} \cdot \mathbf{k}_{\theta}^{\mathrm{F}} \cdot \mathbf{H}_{\theta \mathrm{q}}^{\mathrm{F}}-\left(\mathbf{J}_{\mathrm{q}}^{\mathrm{T}}+\mathbf{H}_{\mathrm{q} \theta}^{\mathrm{F}} \cdot \mathbf{k}_{\theta}^{\mathrm{F}} \cdot \mathbf{J}_{\theta}^{\mathrm{T}}\right) \cdot \mathbf{K}_{C}^{0(\mathrm{~F})} \cdot\left(\mathbf{J}_{\mathrm{q}}+\mathbf{J}_{\theta} \cdot \mathbf{k}_{\theta}^{\mathrm{F}} \cdot \mathbf{H}_{\theta \mathrm{q}}^{\mathrm{F}}\right)\right)^{-1} \cdot\left(\mathbf{J}_{\mathrm{q}}^{\mathrm{T}}+\mathbf{H}_{\mathrm{q} \theta}^{\mathrm{F}} \cdot \mathbf{k}_{\theta}^{\mathrm{F}} \cdot \mathbf{J}_{\theta}^{\mathrm{T}}\right) \cdot \mathbf{K}_{C}^{0(F)}
$$

Similarly, the matrix $\mathbf{K}_{\mathrm{C} \theta}$ can be expressed analytically as

$$
\mathbf{K}_{\mathrm{C} \theta}=\mathbf{k}_{\theta}^{\mathrm{F}} \cdot \mathbf{J}_{\theta}^{\mathrm{T}} \cdot \mathbf{K}_{\mathrm{C}}+\mathbf{k}_{\theta}^{\mathrm{F}} \cdot \mathbf{H}_{\theta \mathrm{q}}^{\mathrm{F}} \cdot \mathbf{K}_{\mathrm{Cq}}
$$

Hence, the developed technique allows us to compute the static equilibrium configuration and Cartesian stiffness matrix for serial chains with external and internal loading applied to the end-effector ad to the node-points (auxiliary loading). It allows us to compute values of the internal variables corresponding to the equilibrium, to obtain the non-linear force-deflection relation and to compute the related stiffness matrices.

\section{Stiffness model of parallel manipulators with internal and external loadings}

The non-linear stiffness modeling technique proposed in Sections 3 deals with separate kinematic chains. In order to be applied to the parallel manipulators, it should be extended by appropriate stiffness model aggregation routines. Hence, it is reasonable to propose the method of aggregation of the elastostatic models of separate kinematic chains to the stiffness model of the parallel manipulator. In general, these routines have to be applicable for both perfect and non-perfect kinematic chains. Besides, it is required to develop numerical algorithms for computing both direct and inverse force-deflection relations that are referred below to as non-linear stiffness and compliance models respectively. 


\subsection{Stiffness model aggregation technique for perfect serial chains}

Let us assume that a parallel manipulator may be presented as a strictly parallel system of the actuated serial legs connecting the base and the end-platform (Figure 5) [66]. Using the methodology described in the previous sections and applying it to each leg, a set of $m$ Cartesian stiffness matrices $\mathbf{K}_{\mathrm{C}}^{(i)}$ expressed with respect to the same coordinate system but corresponding to different platform points can be computed. If initially the chain stiffness matrices have been computed in local coordinate systems, their transformation is performed in a standard way [19], as

$$
\mathbf{K}_{\mathrm{C}}^{\text {glob }}=\left[\begin{array}{cc}
\mathbf{R} & \mathbf{0} \\
\mathbf{0} & \mathbf{R}
\end{array}\right] \cdot \mathbf{K}_{\mathrm{C}}^{\text {loc }} \cdot\left[\begin{array}{cc}
\mathbf{R}^{\mathrm{T}} & \mathbf{0} \\
\mathbf{0} & \mathbf{R}^{\mathrm{T}}
\end{array}\right]
$$

where $\mathbf{R}$ is a $3 \times 3$ rotation matrix describing orientation of the local coordinate system with respect to the global one.

To aggregate these matrices $\mathbf{K}_{\mathrm{C}}^{(i)}$, they must be also re-computed with respect to the same reference point of the platform. Assuming that the platform is rigid enough (as compared to the compliance of the legs), this conversion can be performed by extending the legs by a virtual rigid link connecting the end-point of the leg and the reference point of the platform (see Figure 5 where these extensions are defined by the vectors $\mathbf{v}_{i}$ ).
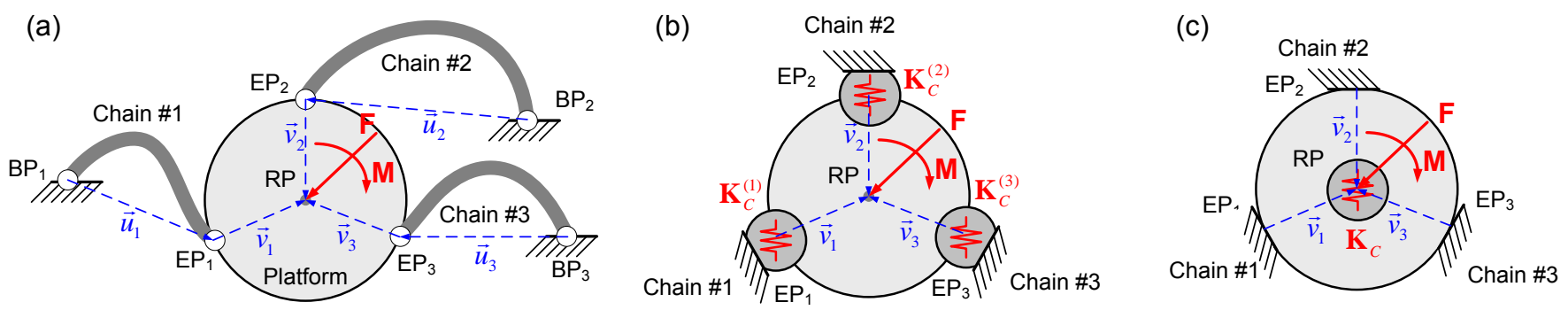

Figure 5 Typical parallel manipulator (a) and transformations of its VJM models (b, c) $\left(\mathrm{EP}_{\mathrm{i}}\right.$ is $i$ th end-point, $\mathrm{BP}_{\mathrm{i}}$ is $i$ th base point, $\mathrm{RP}$ is the reference point, $\left.\vec{u}_{i}=E P_{i}-B P_{i}, \vec{v}_{i}=R P-E P_{i}\right)$

After such extension, an equivalent stiffness matrix of the leg may be expressed using relevant expression for a usual serial chain, i.e. as $\mathbf{J}_{\mathrm{v}}^{(i)-\mathrm{T}} \cdot \mathbf{K}_{\mathrm{C}}^{(i)} \cdot \mathbf{J}_{\mathrm{v}}^{(i)-1}$, where the Jacobian $\mathbf{J}_{\mathrm{v}}^{(i)}$ defines differential relation between the coordinates of the $i$-th virtual spring and the reference frame of the end-platform. Hence, the final expression for the stiffness matrix of the considered parallel manipulator can be written as

$$
\mathbf{K}_{\mathrm{C}}^{(m)}=\sum_{i=1}^{m}\left(\mathbf{J}_{\mathrm{v}}^{(i)^{-\mathrm{T}}} \cdot \mathbf{K}_{\mathrm{C}}^{(i)} \cdot \mathbf{J}_{\mathrm{v}}^{(i)^{-1}}\right)
$$

where $m$ is the number of serial kinematic chains in the manipulator architecture.

As a result, expression (54) allows us to compute Cartesian stiffness matrix for the parallel manipulator based on stiffness matrices for serial chains and transformation Jacobians $\mathbf{J}_{\mathrm{v}}^{(i)}$, which define geometrical mapping between end-points of serial chains and reference point frame (end-effector). Moreover, it is implicitly assumed here that all stiffness matrices (both for the serial chains and for the whole manipulator) are expressed in the same global coordinate system, otherwise they should be recomputed to requisite coordinates. Hence, the axes of all virtual springs are parallel to the axes $x, y, z$ of this system. This allows to evaluate Jacobians $\mathbf{J}_{\mathrm{v}}^{(i)}$ and their inverses from the geometry of the end-platform analytically

$$
\mathbf{J}_{\mathrm{v}}^{(i)}=\left[\begin{array}{cc}
\mathbf{I}_{3} & \left(\mathbf{v}_{i} \times\right) \\
\mathbf{0} & \mathbf{I}_{3}
\end{array}\right]_{6 \times 6}, \quad \mathbf{J}_{\mathrm{v}}^{(i)-1}=\left[\begin{array}{cc}
\mathbf{I}_{3} & -\left(\mathbf{v}_{i} \times\right) \\
\mathbf{0} & \mathbf{I}_{3}
\end{array}\right]_{6 \times 6}
$$

where $\mathbf{I}_{3}$ is $3 \times 3$ identity matrix, and $(\mathbf{v} \times)$ is a skew-symmetric matrix corresponding to the vector $\mathbf{v}$ :

$$
(\mathbf{v} \times)=\left[\begin{array}{ccc}
0 & -v_{z} & v_{\mathrm{y}} \\
v_{\mathrm{z}} & 0 & -v_{\mathrm{x}} \\
-v_{\mathrm{y}} & v_{\mathrm{x}} & 0
\end{array}\right]
$$

Therefore, expression (54) represents explicit aggregation of the leg stiffness matrices with respect to any given reference point of the platform. It is worth mentioning that in practice, the matrices $\mathbf{K}_{\mathrm{C}}^{(i)}$ are always singular while the aggregation usually produces non-singular matrix. Relevant examples are presented in the following sub-section. 


\subsection{Stiffness model aggregation technique for non-perfect serial chains (under internal loadings)}

In the previous Section, it was implicitly assumed that kinematic chains of the parallel manipulator are "perfect", i.e. their geometrical parameters are strictly nominal and their end-frames can be aligned and matched without additional efforts and/or internal stresses, while assembling. However, in practice, the kinematic chain geometry may differ from the nominal one (i.e. be "non-perfect"), and the assembling causes internal stresses and shifting of the end-points location. Thus, let us extend the above aggregation procedure to the case of non-perfect serial chains and develop a technique, which is able to evaluate deflections and internal forces/torques caused by geometrical errors in the chains.

Let us consider a parallel manipulator, which may be presented as a strictly parallel system of the actuated serial legs connecting the fixed base and mobile end-platform [66]. Using the methodology described in the previous sections, each $i$-th leg (serial chain) may be characterized by the geometrical function $\mathbf{t}_{i}=\mathbf{g}_{i}\left(\mathbf{q}_{i}, \boldsymbol{\theta}_{i}\right)$, where $\mathbf{t}_{i}$ defines the end-frame location and $\mathbf{q}_{i}, \boldsymbol{\theta}_{i}$ are passive and virtual joint coordinates respectively, which define kinematic chain configuration. For perfect kinematic chains, the joint coordinates $\mathbf{q}_{i}, \boldsymbol{\theta}_{i}$ are computed using nominal geometrical model of the $\operatorname{leg} \mathbf{g}_{i}^{0}\left(\mathbf{q}_{i}, \boldsymbol{\theta}_{i}\right)$, for the given end-platform location $\mathbf{t}^{0}$. These notations are illustrated by Figure 6a where $A_{i}$ correspond to the end-points of the perfect chains and $O$ is the platform reference point, the vectors $\overline{A_{i} O}$ are denoted as $\mathbf{v}_{i}$. Using the above presented technique, it is possible to compute the Cartesian stiffness matrices of the chains and express them with respect to the same reference point $O$. Let us denote this matrix as $\mathbf{K}_{\mathrm{c}}^{(i)}$.

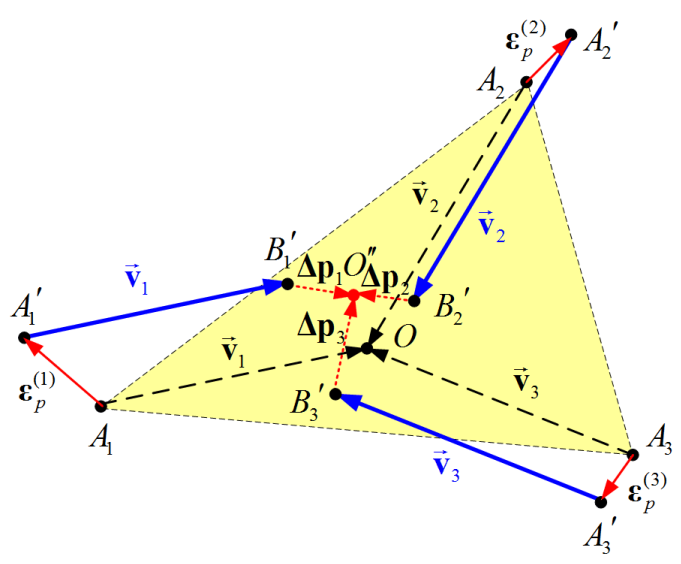

(a) platform before assembling

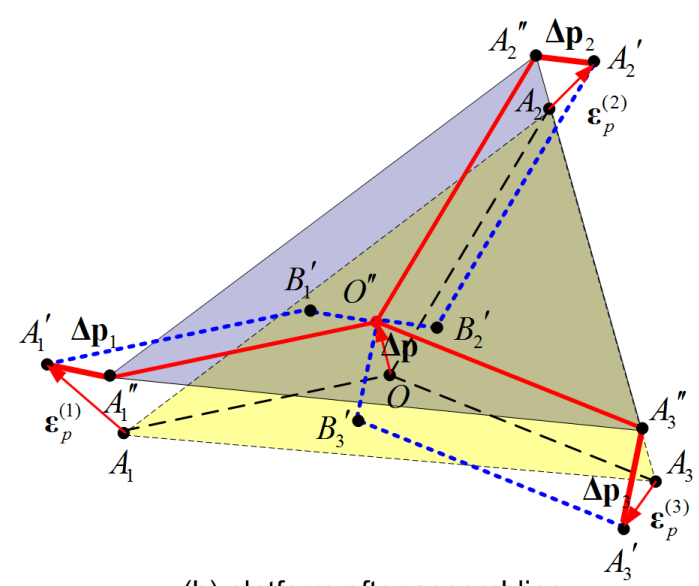

(b) platform after assembling

Figure 6 Transformation of characteristic points of serial chains in assembling of non-perfect parallel manipulator; $\left(A_{i}, A_{i}^{\prime}-\right.$ end-point locations of serial chain before assembling for perfect and non-perfect manipulators respectively, $A_{i}{ }^{\prime \prime}$ - end-point location of serial chain after assembling for non-perfect manipulator)

For the non-perfect chain before assembling, similar configurations $\mathbf{q}_{i}, \boldsymbol{\theta}_{i}$ produce slightly different end-point locations of the chains $\mathbf{t}_{i}=\mathbf{g}_{i}^{0}\left(\mathbf{q}_{i}, \boldsymbol{\theta}_{i}\right)+\boldsymbol{\varepsilon}_{i}$, which are denoted as $A_{i}^{\prime}$ in Figure 6a. Correspondently, assuming that the platform is rigid enough, the points $A_{i}^{\prime}$ can be mapped to $B_{i}^{\prime}$ that differ from $O$ by $\varepsilon_{i}$. Hence, geometrical errors do not allow assembling parallel manipulator at the original reference point.

To assemble all chains in the same reference point, it is required to apply additional efforts. Geometrically, it leads to relocation of the frames corresponding to the points $B_{i}^{\prime}$ to a new position $B_{i}^{\prime \prime}$, and relocations of the point $A_{i}{ }^{\prime}$ to $A_{i}{ }^{\prime \prime}$ that is defined by the vector $\Delta \mathbf{t}_{i}$. In Figure 6b, relevant reference point of the platform is denoted as $O^{\prime \prime}$. Hence, the stiffness model aggregation problem is reduced to computing of a new end-platform location $\mathbf{t}^{0}+\Delta \mathbf{t}$ for which end-frames of all kinematic chains are aligned and matched. Consequently, a manipulator will occupy the most advantageous configuration with respect to the potential energy of the elastic elements.

To compute the end-platform deflection $\Delta \mathbf{t}$, let us assume that the geometrical errors are small enough and corresponding shifting of the chain end-points and end-effector do not change Cartesian stiffness matrices $\mathbf{K}_{\mathrm{C}}^{(i)}$ or their influences are negligible. So, the stiffness matrices of the serial chains $\mathbf{K}_{\mathrm{C}}^{(i)}$ are the same at the points $O, B_{i}{ }^{\prime}$ and $O^{\prime \prime}$ and computed for the nominal configurations $\mathbf{q}_{i}, \boldsymbol{\theta}_{i}$. Let us also assume that the point $O^{\prime \prime}$ is shifted from $O$ by $\Delta \mathbf{p}$ and the mobile platform orientations for the parallel manipulator with perfect and non-perfect serial chains differ by $\Delta \varphi$. This allows us to express the potential energy of the parallel manipulator with geometrical errors in kinematic chains as 


$$
E=\frac{1}{2} \sum_{i=1}^{m}\left(\left(\boldsymbol{\varepsilon}_{i}-\Delta \mathbf{t}\right)^{\mathrm{T}} \cdot \mathbf{K}_{\mathrm{C}}^{(i)} \cdot\left(\boldsymbol{\varepsilon}_{i}-\Delta \mathbf{t}\right)\right)
$$

where $\Delta \mathbf{t}=(\Delta \mathbf{p}, \Delta \boldsymbol{\varphi})$ is displacement (position and orientation) of the reference point.

It is obvious that after assembling, the considered mechanical system will occupy the most advantageous configuration with respect to the potential energy, i.e. $E \rightarrow \min$. It means that the desired vector $\Delta \mathbf{t}$ can be found from the expression

$$
\frac{\partial E}{\partial \Delta \mathbf{t}}=\sum_{i=1}^{m}\left(\mathbf{K}_{\mathrm{C}}^{(i)} \cdot\left(\Delta \mathbf{t}-\boldsymbol{\varepsilon}_{i}\right)\right)=0
$$

that yields the following linear equation

$$
\sum_{i=1}^{m} \mathbf{K}_{\mathrm{C}}^{(i)} \cdot \Delta \mathbf{t}=\sum_{i=1}^{m}\left(\mathbf{K}_{\mathrm{C}}^{(i)} \cdot \boldsymbol{\varepsilon}_{i}\right)
$$

which allows us to evaluate the end-platform deflection

$$
\Delta \mathbf{t}=\left(\sum_{i=1}^{m} \mathbf{K}_{\mathrm{C}}^{(i)}\right)^{-1} \cdot \sum_{i=1}^{m}\left(\mathbf{K}_{\mathrm{C}}^{(i)} \cdot \boldsymbol{\varepsilon}_{i}\right)
$$

and the end-platform location after assembling

$$
\mathbf{t}^{\prime}=\mathbf{t}^{0}+\Delta \mathbf{t}
$$

For each separate kinematic chain, the end-frame deflections due to assembling can be expressed as

$$
\Delta \mathbf{t}_{i}=\Delta \mathbf{t}-\boldsymbol{\varepsilon}_{i}=\left(\sum_{i=1}^{m} \mathbf{K}_{\mathrm{C}}^{(i)}\right)^{-1} \cdot \sum_{i=1}^{m}\left(\mathbf{K}_{\mathrm{C}}^{(i)} \cdot \boldsymbol{\varepsilon}_{i}\right)-\boldsymbol{\varepsilon}_{i}
$$

This allows us to compute the loading for each kinematic chain applied to the end-point (due to interaction with other non-perfect chains)

$$
\mathbf{F}_{i}=\mathbf{K}_{\mathrm{C}}^{(i)} \cdot \Delta \mathbf{t}_{i}
$$

and corresponding loadings in the virtual joints $\boldsymbol{\tau}_{\theta}^{(i)}$

$$
\boldsymbol{\tau}_{\theta}^{(i)}=\mathbf{J}_{\theta}^{(i) \mathrm{T}} \cdot \mathbf{F}_{i}=\mathbf{J}_{\theta}^{(i) \mathrm{T}} \cdot \mathbf{K}_{\mathrm{C}}^{(i)} \cdot \Delta \mathbf{t}_{i}
$$

It is worth mentioning that here $\sum_{i=1}^{m} \mathbf{F}_{i}=\mathbf{0}$, since there is no external loading applied to the platform reference point after the assembling. Besides, it is possible to compute relevant deflections of the virtual and passive joint coordinates of the chains

$$
\boldsymbol{\theta}_{i}=\mathbf{K}_{\mathrm{C} \theta}^{(i)} \cdot \Delta \mathbf{t}_{i} ; \quad \Delta \mathbf{q}_{i}=\mathbf{K}_{\mathrm{Cq}}^{(i)} \cdot \Delta \mathbf{t}_{i}
$$

caused by the assembling.

Thus, the above expressions allow us to evaluate the end-platform deflection and internal forces/torques caused by assembling of kinematic chains with geometrical errors. However, the total manipulator Cartesian stiffness matrix $\mathbf{K}_{\mathrm{C}}$ is assumed to be the same as in the case of perfect serial chains, since the geometrical errors are assumed to be small enough. It should be mentioned that the difference in the potential energy due to the geometrical errors of serial chains is the second order of smallness, as follows from eq. (57). On the other hand, this non-perfection leads to the errors in the end-effector position and orientation with the first order of smallness. For this reason, the geometrical errors from manufacturing and assembling do not appear directly in the final expression for the stiffness matrix (50). Nevertheless, the above mentioned deviations effect the equilibrium configuration with respect to which the Jacobians and the stiffness coefficients are computed. For this reason, the authors prefer to take into account such deviations of the second order smallness in order to detect some non-linear phenomenon in the manipulator behaviour, such as geometrical buckling studied in our previous paper [10].

Hence, here the stiffness model assembling technique from Section 4.1 has been extended for the case of parallel manipulators with geometrical errors in serial chains. In addition to computing of aggregated Cartesian stiffness matrix, it allows us to evaluate internal deflections and forces/torques in joints, as well as deflections of the reference point caused by geometrical errors in kinematic chains. In spite of this issue has essential practical significance for evaluating the desired tolerances in links/joints geometry and corresponding internal stresses in over-constrained mechanisms, it has never been studied before. 


\subsection{Stiffness model of non-perfect parallel manipulator under external loading}

Let us focus on the aggregation of stiffness models of separate serial chains into the stiffness model of the whole parallel manipulator in the loaded mode. To solve this problem, it is necessary to obtain the non-linear force-deflection relation, which takes into account elastostatic properties of all kinematic chains, and to compute corresponding Cartesian stiffness matrix.

Let us assume that the end-points of all kinematic chains are aligned and matched in the same target point $\mathbf{t}_{0}$, which corresponds to the desired end-platform location. This point is assumed to be known and allows us to compute, from the inverse kinematic model, the actuator and passive joint coordinates defining nominal configurations of the chains $\left(\mathbf{q}_{0 i}, \boldsymbol{\theta}_{0 i}\right)$. It is also assumed that the stiffness models of all kinematic chains have been already obtained using techniques proposed in Sections 4.2 and are presented in the form of partial non-linear force-deflection relations $\mathbf{F}_{i}=f_{i}\left(\mathbf{t} \mid \mathbf{t}_{0}\right)$ corresponding to the target point $\mathbf{t}_{0}$.

It is evident that the external loading $\mathbf{F}$ changes the end-platform location $\mathbf{t}_{0}$, hence it is reasonable to consider the set of locations $\mathbf{t}$ in the neighborhood of target one. Under the above assumptions, for any given point $\mathbf{t}$ from neighborhood of $\mathbf{t}_{0}$ it is possible to compute both the partial forces $\mathbf{F}_{i}$ and corresponding equilibrium configurations $\left(\mathbf{q}_{i}, \boldsymbol{\theta}_{i}\right)$. Then, in accordance with the superposition principle, the desired non-linear force-deflection relation for the whole parallel manipulator can be found by straightforward summation of all partial forces $\mathbf{F}_{i}$, i.e.

$$
\mathbf{F}=\sum_{i=1}^{m} f_{i}\left(\mathbf{t} \mid \mathbf{t}_{0}\right)
$$

where $\mathbf{F}$ denotes the total external loading applied to the end-platform. As a result, corresponding curves can be obtained by multiple repetition of the above described procedures for different values of the end-platform location $\mathbf{t}$.

Furthermore, for each given $\mathbf{t}$, the stiffness matrices $\mathbf{K}_{\mathrm{C}}^{(i)}$ of all kinematic chains can be computed using expression (50). This allows us to compute the Cartesian stiffness matrix $\mathbf{K}_{\mathrm{C}}$ of the whole parallel manipulator as a sum

$$
\mathbf{K}_{\mathrm{C}}=\sum_{i=1}^{m} \mathbf{K}_{\mathrm{C}}^{(i)}
$$

However, the matrices $\mathbf{K}_{\mathrm{Cq}}^{(i)}$ and $\mathbf{K}_{\mathrm{C} \theta}^{(i)}$ defining the "sensitivity" of the chain joint coordinates ( $\left.\mathbf{q}_{i}, \boldsymbol{\theta}_{i}\right)$ to the end-platform displacement cannot be aggregated in this way, they should be used separately to evaluate stresses in joints/links and resistance of the chain configurations with respect to external loading $\mathbf{F}$

$$
\boldsymbol{\tau}_{\theta}^{(i)}=\mathbf{J}_{\theta}^{(i) \mathrm{T}} \cdot \mathbf{K}_{\mathrm{C}}^{(i)} \cdot\left(\mathbf{t}-\mathbf{t}_{0}\right) ; \quad \delta \mathbf{q}_{i}=\mathbf{K}_{\mathrm{Cq}}^{(i)} \cdot\left(\mathbf{t}-\mathbf{t}_{0}\right) ; \quad \delta \boldsymbol{\theta}_{i}=\mathbf{K}_{\mathrm{C} \theta}^{(i)} \cdot\left(\mathbf{t}-\mathbf{t}_{0}\right)
$$

where $\mathbf{J}_{\theta}^{(i)}$ is Jacobian matrix of $i$-th kinematic chain with respect to virtual joint coordinates.

It is worth mentioning that above it was implicitly assumed that the manipulator assembling is equivalent to the aligning and matching of the chain end-frames. To deal with more general case, when the chains are connected to the different points of the platform, it is necessary to slightly modify the chain geometrical models and to re-compute the forces/torques and the stiffness matrices by adding a virtual rigid link connecting the end-point of the chain and the reference point of the platform (see Figure 5 where these extensions are defined by the vectors $\mathbf{v}_{i}$ ). After the relevant transformations, the above presented technique can be applied straightforwardly.

Besides, here there are no evident differences in stiffness models aggregation of perfect and non-perfect kinematic chains. However, here the chain geometrical errors are implicitly included in the functions $\mathbf{g}_{i}^{\varepsilon}\left(\mathbf{q}_{i}, \boldsymbol{\theta}_{i}\right)$. In particular for non-perfect chains, it is assumed that the nominal values of the joint coordinates $\left(\mathbf{q}_{0 i}, \boldsymbol{\theta}_{0 i}\right)$ produce the end-point location vector which differs from $\mathbf{t}_{0}$ :

$$
\mathbf{g}_{i}^{\varepsilon}\left(\mathbf{q}_{0 i}, \boldsymbol{\theta}_{0 i}\right)=\mathbf{t}_{0}+\boldsymbol{\varepsilon}_{i}
$$

where $\varepsilon_{i}$ accumulates influences of all geometrical errors on the end-point location of $i$-th chain. As a result, the end-platform cannot be located in the target point $\mathbf{t}_{0}$ without external loading, i.e.

$$
\left.\sum_{i=1}^{m} f_{i}\left(\mathbf{t} \mid \mathbf{t}_{0}\right)\right|_{\mathbf{t}=\mathbf{t}_{0}} \neq \mathbf{0}
$$

Moreover, without external loading, the end-platform location $\mathbf{t}_{\varepsilon}$ is different from the target one $\mathbf{t}_{0}$. The vector $\mathbf{t}_{\varepsilon}$ can be computed from the equation

$$
\sum_{i=1}^{m} f_{i}\left(\mathbf{t}_{\varepsilon} \mid \mathbf{t}_{0}\right)=\mathbf{0}
$$




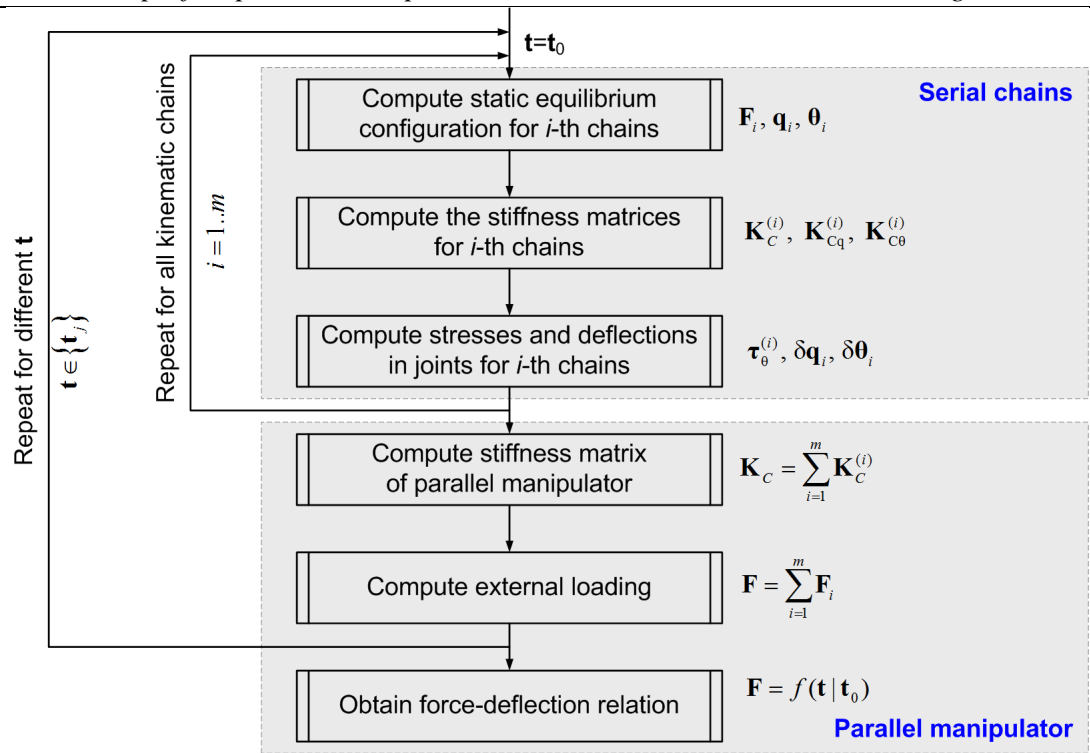

Figure 7 Aggregation of serial chains stiffness models technique

which will be considered in the next sub-section. Corresponding internal forces $\mathbf{F}_{i}^{\varepsilon}$ defining the chain loadings due to the geometrical errors in the chains can be computed by simple substitution $\mathbf{t}_{\varepsilon}$ to the partial force deflection relations

$$
\mathbf{F}_{i}^{\varepsilon}=\left.f_{i}\left(\mathbf{t} \mid \mathbf{t}_{0}\right)\right|_{\mathbf{t}=\mathbf{t}_{e}}
$$

It is obvious that the sum of the $\mathbf{F}_{i}^{\varepsilon}$ is equal to zero but they produce stresses in the links and joints if the parallel manipulator is over-constrained.

Hence, the developed aggregation technique allows us to obtain the non-linear force-deflection relation for a parallel manipulator in the loaded mode as well as to compute Cartesian stiffness matrices for any given target point $\mathbf{t}_{0}$ and given set of the end-point locations $\{\mathbf{t}\}$. This technique is summarized in Figure 7.

\subsection{Model inversion: compliance properties of parallel manipulator under the loading}

The non-linear force-deflection relation (66) allows us to evaluate the external force/torque $\mathbf{F}$ required to locate the manipulator in the target point $\mathbf{t}$ (assuming that the actuated coordinates are computed for the end-platform location $\mathbf{t}_{0}$ corresponding to the unloaded configuration). However in practice, it is often necessary to determine the end platform resistance to the external loading, i.e. to compute the deflection caused by the force $\mathbf{F}$ applied to the end-platform. The desired value can be found from the non-linear compliance model that in general case is expressed as

$$
\mathbf{t}=f^{-1}\left(\mathbf{F} \mid \mathbf{t}_{0}\right)
$$

and is defined by the inverse $f^{-1}(\ldots)$ which for parallel manipulators usually exists (due to over-constrained structure). In contrast, for serial chains with passive joints, the function $f^{-1}(\ldots)$ cannot be computed since the corresponding Cartesian stiffness matrix $\mathbf{K}_{\mathrm{C}}^{(i)}$ is singular.

It is obvious that in a general case, the function $f^{-1}(\ldots)$ cannot be expressed analytically. Hence, it is required that a dedicated iterative procedure, which is able to solve the non-linear equation (73) for $\mathbf{t}$ (assuming that $\mathbf{F}$ is given). It is proposed here to apply a modification of Newton-Raphson technique which iteratively updates the desired value $\mathbf{t}$ in accordance with the expression

$$
\mathbf{t}^{\prime}=\mathbf{t}+\mathbf{K}_{\mathrm{C}}^{-1}\left(\mathbf{t} \mid \mathbf{t}_{0}\right) \cdot\left(\mathbf{F}-f\left(\mathbf{t} \mid \mathbf{t}_{0}\right)\right)
$$

where $\mathbf{t}^{\prime}$ corresponds to the next iteration, $\mathbf{K}_{\mathrm{C}}\left(\mathbf{t} \mid \mathbf{t}_{0}\right)$ is the Cartesian stiffness matrix computed in the point $\mathbf{t}$, and $\mathbf{t}_{0}$ denotes the unloaded location of the end-platform. For this iterative scheme, $\mathbf{t}_{0}$ can be also used as the initial value of $\mathbf{t}$. Similar to sub-section 4.5.1, within each iterative loop, corresponding configurations $\left(\mathbf{q}_{i}, \boldsymbol{\theta}_{i}\right)$, the loadings $\mathbf{F}_{i}$ and stiffness matrices $\mathbf{K}_{\mathrm{C}}^{(i)}$ for each kinematic chain are computed using equations (36), (50). 


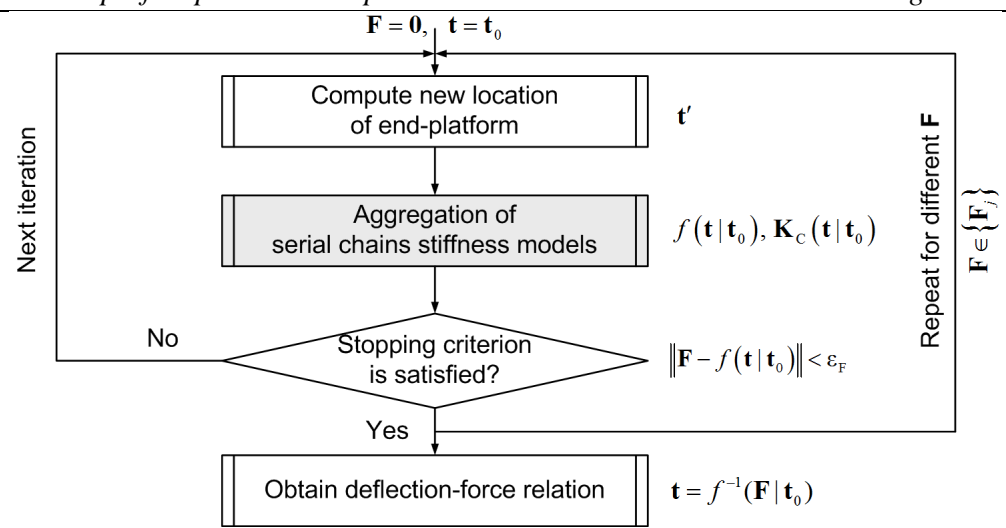

Figure 8 Procedure for obtaining deflection-force relation for loaded parallel manipulator

As it follows from the relevant study, convergence of this iterative procedure is good enough if the function $f(\ldots)$ is smooth and non-singular in the neighborhood of $\mathbf{t}_{0}$. If it is required to improve convergence, it is possible to apply the same technique as in Section 3.3, when the force $\mathbf{F}$ is modified from iteration to iteration in accordance with the expression $\mathbf{F}^{\prime}=\alpha \cdot \mathbf{F}$, where a scalar variable $\alpha$ is monotonically increasing from 0 up to 1 . The stopping criterion can be expressed in a straightforward way as

$$
\left\|\mathbf{F}-f\left(\mathbf{t} \mid \mathbf{t}_{0}\right)\right\|<\varepsilon_{\mathrm{F}}
$$

where $\varepsilon_{\mathrm{F}}$ is the desired accuracy. More details presentation of the developed iterative routines is given in Figure 8

Summarizing this sub-section, it is worth mentioning that the developed technique allows to obtain the desired non-linear deflection-force relation describing the end-platform resistance with respect to the external force for a given $\mathbf{t}_{0}$, which corresponds to the unloaded (nominal) manipulator configuration. The above presented results can be used both for stiffness modeling of manipulator under the loading and for compliance error compensation.

\section{Application examples: parallel translational manipulator}

\subsection{Non-perfect parallel manipulator without external loading}

Let us illustrate the developed stiffness model aggregation technique by examples that deal with assembling of Orthoglide parallel translational manipulator with geometrical errors in kinematic chains (Figure 9). The manipulator consist of three kinematic chains with one translational actuator along the axes $\mathrm{x}$, y or $\mathrm{z}$, two passive U-joints (or two separate rotational joints) and kinematic parallelogram between them. It should be mentioned that two chains with kinematic parallelograms would be sufficient to restrict the motion in translation. However in order to increase the manipulator stiffness, the third actuated chain has been introduced that impose redundant constraints on the mobile platform, i.e. the Orthoglide manipulator has an over-constrained structure. This manipulator has the working area of size $200 \times 200 \times 200 \mathrm{~mm}$ with the transmission factor from 0.5 to 2 ; the detailed geometrical parameters are presented in [58], the link stiffness matrices were computed using an approach developed in [34], where their numerical values are presented as well. Let us assume that the manipulators have geometrical errors in the kinematic chains, which have effects on the end-point location and provoke internal loadings in the joints.

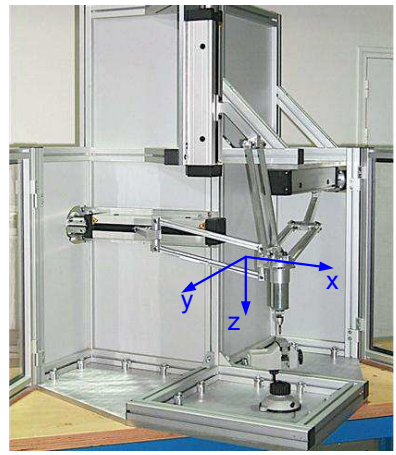

(a) Photo

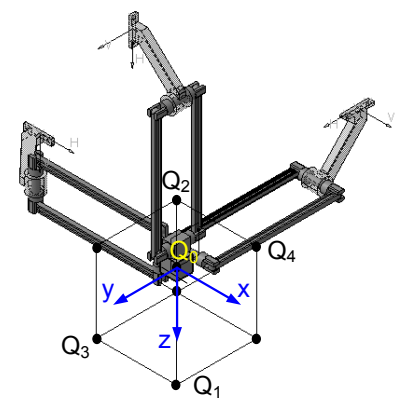

(b) CAD-model

Figure 9 Photo and CAD models of Orthoglide manipulator 
Taking into account the shape of the dexterous workspace, let us focus on the stiffness analysis of these manipulators in five characteristic points: isotropic point $\mathrm{Q}_{0}$, two limit points $\mathrm{Q}_{1}$ and $\mathrm{Q}_{2}$. with symmetrical configuration and two limit points $\mathrm{Q}_{3}$ and $\mathrm{Q}_{4}$. with non-symmetrical configuration [74] (see Figure 9). Let us estimate the end-effector location and internal deflections/loadings caused by the geometrical errors in the chains. The stiffness matrices of the chains for the manipulator in the points $\mathrm{Q}_{0} \ldots \mathrm{Q}_{4}$ have been computed using the technique proposed in Section 3.3.

For illustration purposes, let us investigate two types of geometrical errors

Case A: Each actuator of the manipulator has a position error $1 \mathrm{~mm}$ in actuator location $\boldsymbol{\varepsilon}_{1}^{0}=(1,0,0,0,0,0)^{T} ; \quad \boldsymbol{\varepsilon}_{2}^{0}=(0,1,0,0,0,0)^{T} ; \quad \boldsymbol{\varepsilon}_{3}^{0}=(0,0,1,0,0,0)^{T}$

Case B: $\quad$ Each actuator of the manipulator has an angular error $1^{\circ}$ in actuator location $\boldsymbol{\varepsilon}_{1}^{0}=\left(0,0,0,1^{\circ}, 0,0\right)^{T} ; \quad \boldsymbol{\varepsilon}_{2}^{0}=\left(0,0,0,0,1^{\circ}, 0\right)^{T} ; \quad \boldsymbol{\varepsilon}_{3}^{0}=\left(0,0,0,0,0,1^{\circ}\right)^{T}$.

It should be mentioned that the developed approach can be applied for the manipulator of general architecture and non-regular structure of vectors $\boldsymbol{\varepsilon}_{i}^{0}$. However here, the regular errors were used to show that the manufacturing and assembling deviations effect the manipulator end-platform location and it Cartesian stiffness matrix

In case $\mathrm{A}$, as it follows from the chains geometry, the deflections of the chain end-points before assembling $\boldsymbol{\varepsilon}_{i}$ may be expressed as $\boldsymbol{\varepsilon}_{1}=\boldsymbol{\varepsilon}_{1}^{0} ; \boldsymbol{\varepsilon}_{2}=\boldsymbol{\varepsilon}_{2}^{0} ; \boldsymbol{\varepsilon}_{3}=\boldsymbol{\varepsilon}_{3}^{0}$. In case B, the values $\boldsymbol{\varepsilon}_{i}$ should be computed using the geometrical model $\mathbf{g}_{i}\left(\mathbf{q}_{i}, \boldsymbol{\theta}_{i}\right)$, relevant computational results are summarized in Table 3 .

Table 3 Equivalent end-point errors caused by inaccuracy in serial chains for three chains of Orhoglide manipulator

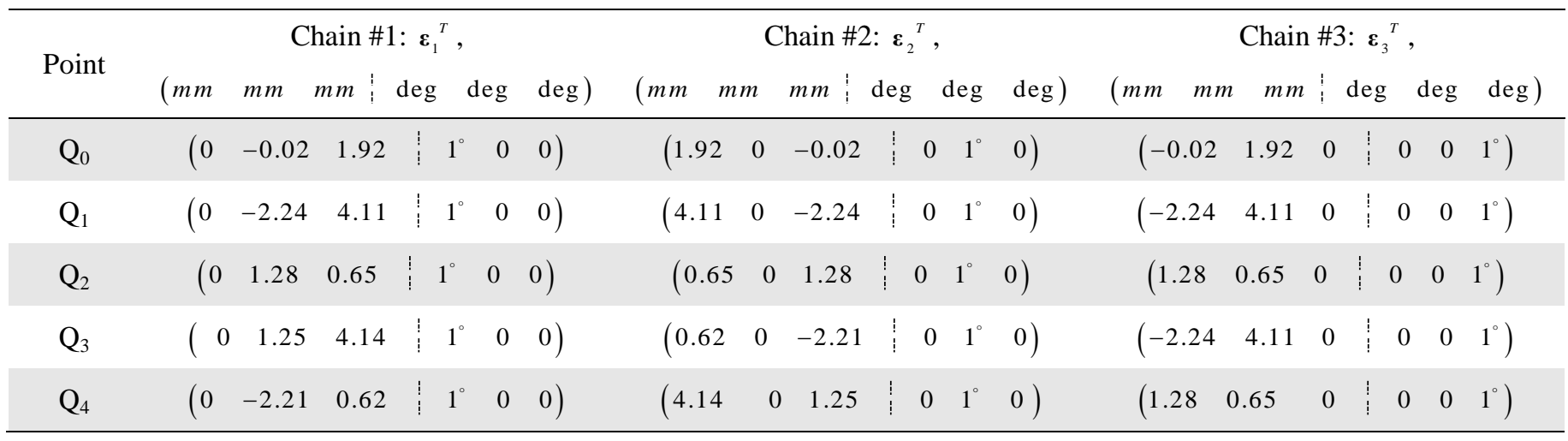

Further, substituting deflections $\boldsymbol{\varepsilon}_{i}$ and corresponding chain stiffness matrices $\mathbf{K}_{\mathrm{C}}^{(i)}$ into formulas (60)-(65), were computed the assembling-induced values of the end-platform displacement, the internal forces/torques and the relevant displacements in virtual and passive joints. The main results of this study are summarized in Tables 4-5, where $\Delta q^{\max }$ is the maximum rotational displacement of passive joints, $\theta_{\mathrm{p}}^{\max }, \theta_{\varphi}^{\max }$ are maximum displacement of translational and rotational virtual springs respectively, $\tau_{\mathrm{p}}^{\max }, \tau_{\varphi}^{\max }$ are maximum torques in translational and rotational virtual joints respectively, $M^{\max }$ is the maximum moment in the chains, caused by assembling of a parallel manipulator with the non-perfect kinematic chains.

These results show that in the Case A (Table 4), the geometrical errors in the kinematic chains do not cause any internal loading. However, they provoke the shift of the end-platform location up to $2.02 \mathrm{~mm}$ (point $\mathrm{Q}_{2}$ ). Corresponding changes in passive joint coordinates are about $0.42^{\circ}$ (point $\mathrm{Q}_{2}$ )

Table 4 Assembling of Orthoglide manipulator with non-perfect chains: loadings and displacements for the case A $\left(\Delta \mathbf{t}=\left[\delta_{1}, \delta_{2}, \delta_{3}, 0,0,0\right]^{T}, \mathbf{F}_{1}=\mathbf{0}, \mathbf{F}_{2}=\mathbf{0}, \mathbf{F}_{3}=\mathbf{0}\right)$

\begin{tabular}{ccccc}
\hline Point & $\begin{array}{c}\text { Displacement } \\
\text { of end-point } \Delta \mathrm{t}\end{array}$ & $\begin{array}{c}\text { Deflections and loadings } \\
\text { in joints and links }\end{array}$ \\
\hline $\mathrm{Q}_{0}$ & $\delta_{1}=\delta_{2}=\delta_{3}=1 \mathrm{~mm} ;$ & $\Delta q^{\max }=0.18^{\circ} ; \quad \theta_{\mathrm{p}}^{\max }=0 ; \quad \theta_{\varphi}^{\max }=0 \quad \tau_{\mathrm{p}}^{\max }=0 ; \quad \tau_{\varphi}^{\max }=0$ \\
$\mathrm{Q}_{1}$ & $\delta_{1}=\delta_{2}=\delta_{3}=0.50 \mathrm{~mm}$ & $\Delta q^{\max }=0.14^{\circ} ; \quad \theta_{\mathrm{p}}^{\max }=0 ; \quad \theta_{\varphi}^{\max }=0 \quad \tau_{\mathrm{p}}^{\max }=0 ; \quad \tau_{\varphi}^{\max }=0$ \\
$\mathrm{Q}_{2}$ & $\delta_{1}=\delta_{2}=\delta_{3}=2.02 \mathrm{~mm} ;$ & $\Delta q^{\max }=0.42^{\circ} ; \quad \theta_{\mathrm{p}}^{\max }=0 ; \quad \theta_{\varphi}^{\max }=0 \quad \tau_{\mathrm{p}}^{\max }=0 ; \quad \tau_{\varphi}^{\max }=0$ \\
$\mathrm{Q}_{3}$ & $\delta_{1}=\delta_{2}=0.73 \mathrm{~mm} ; \delta_{3}=0.27 \mathrm{~mm}$ & $\Delta q^{\max }=0.20^{\circ} ; \quad \theta_{\mathrm{p}}^{\max }=0 ; \quad \theta_{\varphi}^{\max }=0 \quad \tau_{\mathrm{p}}^{\max }=0 ; \quad \tau_{\varphi}^{\max }=0$ \\
$\mathrm{Q}_{4}$ & $\delta_{1}=\delta_{2}=0.56 \mathrm{~mm} ; \delta_{3}=1.28 \mathrm{~mm}$ & $\Delta q^{\max }=0.26^{\circ} ; \quad \theta_{\mathrm{p}}^{\max }=0 ; \quad \theta_{\varphi}^{\max }=0 \quad \tau_{\mathrm{p}}^{\max }=0 ; \quad \tau_{\varphi}^{\max }=0$ \\
\hline
\end{tabular}


Table 5 Assembling of Orthoglide manipulator with non-perfect chains: loadings and displacements for the case B $\left(\Delta \mathbf{t}=\left[\delta_{1}, \delta_{2}, \delta_{3}, \varphi_{1}, \varphi_{2}, \varphi_{3}\right]^{T}, \mathbf{F}_{1} \neq \mathbf{0}, \mathbf{F}_{2} \neq \mathbf{0}, \mathbf{F}_{3} \neq \mathbf{0}\right)$

\begin{tabular}{|c|c|c|}
\hline Point & $\begin{array}{c}\text { Displacement } \\
\text { of end-point } \Delta \mathbf{t}\end{array}$ & $\begin{array}{l}\text { Deflections and loadings } \\
\text { in joints and links }\end{array}$ \\
\hline $\mathrm{Q}_{0}$ & $\begin{array}{l}\delta_{1}=\delta_{2}=\delta_{3}=0 \mathrm{~mm} \\
\varphi_{1}=\varphi_{2}=\varphi_{3}=0.03^{\circ}\end{array}$ & $\begin{array}{lll}M^{\max }=2.09 \mathrm{~N} \cdot \mathrm{m} & \tau_{\mathrm{p}}^{\max }=0 ; & \tau_{\varphi}^{\max }=2.09 \mathrm{~N} \cdot \mathrm{m} ; \\
\Delta q^{\max }=0.31^{\circ} ; & \theta_{\mathrm{p}}^{\max }=0.05 \mathrm{~mm} ; & \theta_{\varphi}^{\max }=0.94^{\circ} ;\end{array}$ \\
\hline $\mathrm{Q}_{1}$ & $\begin{array}{l}\delta_{1}=\delta_{2}=\delta_{3}=0.41 \mathrm{~mm} \\
\varphi_{1}=\varphi_{2}=\varphi_{3}=-0.62^{\circ}\end{array}$ & $\begin{array}{lll}M^{\max }=8.91 \mathrm{~N} \cdot \mathrm{m} & \tau_{\mathrm{p}}^{\max }=0 ; & \tau_{\varphi}^{\max }=11.96 \mathrm{~N} \cdot \mathrm{m} ; \\
\Delta q^{\max }=0.63^{\circ} ; & \theta_{\mathrm{p}}^{\max }=0.54 \mathrm{~mm} ; & \theta_{\varphi}^{\max }=1.74^{\circ} ;\end{array}$ \\
\hline $\mathrm{Q}_{2}$ & $\begin{array}{l}\delta_{1}=\delta_{2}=\delta_{3}=-0.96 \mathrm{~mm} \\
\varphi_{1}=\varphi_{2}=\varphi_{3}=0.21^{\circ}\end{array}$ & $\begin{array}{lll}M^{\max }=1.48 \mathrm{~N} \cdot \mathrm{m} & \tau_{\mathrm{p}}^{\max }=0 ; & \tau_{\varphi}^{\max }=1.75 \mathrm{~N} \cdot \mathrm{m} ; \\
\Delta q^{\max }=0.52^{\circ} ; & \theta_{\mathrm{p}}^{\max }=0.14 \mathrm{~mm} ; & \theta_{\varphi}^{\max }=0.80^{\circ} ;\end{array}$ \\
\hline $\mathrm{Q}_{3}$ & $\begin{array}{lll}\delta_{1}=-0.91 \mathrm{~mm} ; & \delta_{2}=1.31 \mathrm{~mm} ; & \delta_{3}=0.58 \mathrm{~mm} \\
\varphi_{1}=-0.19^{\circ} ; & \varphi_{2}=-0.49^{\circ} ; & \varphi_{3}=0.44^{\circ}\end{array}$ & $\begin{array}{lll}M^{\max }=4.33 \mathrm{~N} \cdot \mathrm{m} ; & \tau_{\mathrm{p}}^{\max }=0 ; & \tau_{\varphi}^{\max }=4.84 \mathrm{~N} \cdot \mathrm{m} ; \\
\Delta q^{\max }=0.67^{\circ} ; & \theta_{\mathrm{p}}^{\max }=0.99 \mathrm{~mm} ; & \theta_{\varphi}^{\max }=1.49^{\circ} ;\end{array}$ \\
\hline $\mathrm{Q}_{4}$ & $\begin{array}{lll}\delta_{1}=0.93 \mathrm{~mm} ; & \delta_{2}=-0.10 \mathrm{~mm} ; & \delta_{3}=-0.25 \mathrm{~mm} ; \\
\varphi_{1}=0.33^{\circ} ; & \varphi_{2}=0.22^{\circ} ; & \varphi_{3}=-0.31^{\circ} ;\end{array}$ & $\begin{array}{lll}M^{\max }=2.98 \mathrm{~N} \cdot \mathrm{m} ; & \tau_{\mathrm{p}}^{\max }=0 ; & \tau_{\varphi}^{\max }=4.0 \mathrm{~N} \cdot \mathrm{m} ; \\
\Delta q^{\max }=0.59^{\circ} ; & \theta_{\mathrm{p}}^{\max }=0.62 \mathrm{~mm} ; & \theta_{\varphi}^{\max }=1.30^{\circ} ;\end{array}$ \\
\hline
\end{tabular}

In contrast, in the Case B, the geometrical errors in the kinematic chains of Orthoglide (Table 5) cause essential internal loadings. For instance, in point $\mathrm{Q}_{1}$ the torque applied to the end-point of the chain can reach up to $8.91 \mathrm{~N} \cdot \mathrm{m}$. This loading induces displacements up to $0.41 \mathrm{~mm}$ and $0.62^{\circ}$ for translational and rotational virtual springs respectively. It should be noted that the loadings for rotational virtual springs are up to $11.96 \mathrm{~N} \cdot \mathrm{m}$, but for translational virtual springs they are equal to zero (in spite of non-zero deflections in them). Nevertheless, this result is reasonable due to the non-diagonal structure of the matrices $\mathbf{K}_{\mathrm{C}}^{(i)}$ representing couplings between rotational and translational deflections. Variations in the passive joint coordinates can reach up to $0.67^{\circ}$ (Point $\mathrm{Q}_{3}$ ). For the end-platform, this case study gives the positional deflection up to $1.31 \mathrm{~mm}\left(\mathrm{Point}_{3}\right)$ and the rotational deflection up to $0.62^{\circ}$ (Point $\mathrm{Q}_{1}$ ). It is obvious that the total sum of all internal loadings is equal to zero. The above presented simulation results show that the developed technique can be used to estimate internal stresses in the manipulator components and to avoid damage of mechanical elements.

\subsection{Non-perfect parallel manipulator under external loading}

Now let us consider the chain stiffness model aggregation of Orthoglide manipulator under external loading caused by groove milling.. According to [42], such technological process causes forces $F_{x}=215 N ; F_{y}=-10 N ; F_{z}=-25 N$. The tool length $h=100 \mathrm{~mm}$ leads to torques at the manipulator end-effector $M_{x},=1 \mathrm{~N} \cdot \mathrm{m}$ and $M_{y},=21.5 \mathrm{~N} \cdot \mathrm{m}$. It should be noted that to define appropriate cutting conditions the frequency analysis is required, which may demand to change the feed rate or cutting depth in order protect the robot and equipment. It is assumed that the manipulator has two sources of inaccuracy:

(i) the assembling errors in the kinematic chains causing internal forces and relevant deflections in joints and links due to manipulator over-constrained structure;

(ii) the external loading $\|\mathbf{F}\|=217 \mathrm{~N}$ caused by the cutting force, which generates essential compliance deflections causing non-desirable end-platform displacement.

Similar to the previous example, it is assumed that the first source of inaccuracy can be caused by translational (Case A) and rotational (Case B) errors in the actuator locations.

Let us consider the case study when Orthoglide performs milling from the point $\mathrm{Q}_{2}$ to $\mathrm{Q}_{5}(-73.65,126.35,-73.65)$ following the straight line. Simulation results for two error sources for the Case A and Case B are presented in Figure 10 and Figure 11 respectively. They include the target trajectories, displacements caused by the cutting forces and non-perfect geometry as well as total compliance errors and the displacement evaluated using the superposition principle. The results are presented for the displacements in $\mathrm{x}$ - and $\mathrm{z}$-directions. 


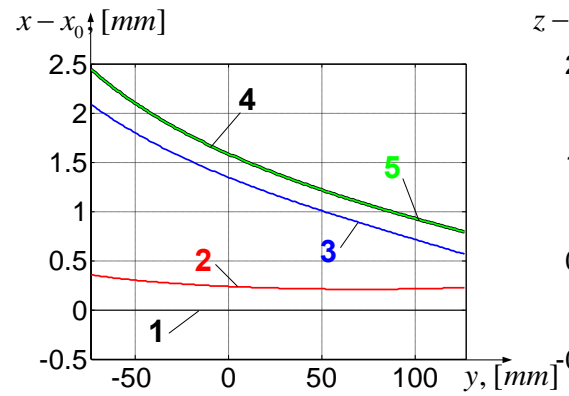

(a) Errors in x-direction

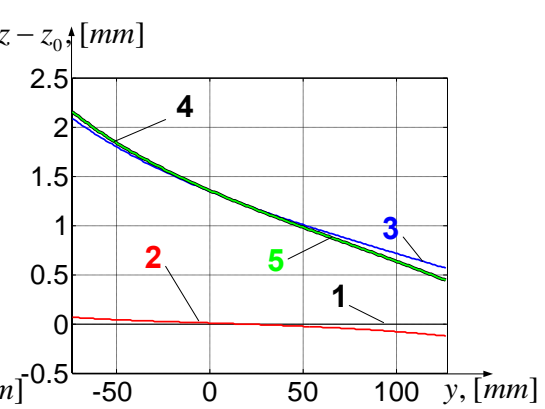

(a) Errors in z-direction

Figure 10 Case A: Displacements caused different sources of inaccuracy during milling along the straight line from point $\mathrm{Q}_{2}$ to $\mathrm{Q}_{4}$ using Orthoglide manipulator: (1) target trajectory, (2) displacements caused by cutting forces, (3) displacements caused by non-perfect geometry, (4) total compliance error, (5) displacements obtained using superposition principle.

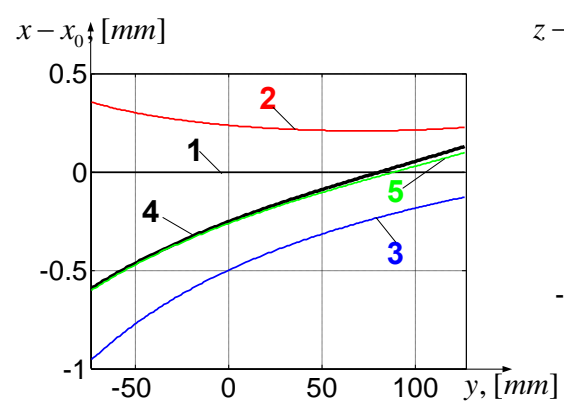

(a) Errors in x-direction

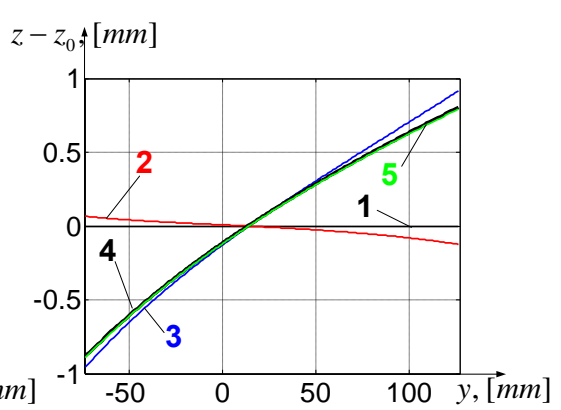

(a) Errors in z-direction

Figure 11 Case B: Displacements caused different sources of inaccuracy during milling along the straight line from point $\mathrm{Q}_{2}$ to $\mathrm{Q}_{4}$ using Orthoglide manipulator: (1) target trajectory, (2) displacements caused by cutting forces, (3) displacements caused by non-perfect geometry, (4) total compliance error, (5) displacements obtained using superposition principle.

The obtained results show that, due to the cutting force, the end-platform displacement in the $\mathrm{x}$-direction with respect to the target trajectory may reach up to $0.4 \mathrm{~mm}$ and its variations are insignificant (compared to the errors caused by inaccuracy in the serial chains). In particular, in Case A, the errors in the $\mathrm{x}$-direction induced by cutting forces and inaccuracy in serial chains have the same direction, and, consequently total error is close to $2.5 \mathrm{~mm}$ in the point $\mathrm{Q}_{2}$ and about $0.8 \mathrm{~mm}$ in the vicinity of the point $\mathrm{Q}_{4}$. In contrast, in the Case $\mathrm{B}$, the compliance errors in the $\mathrm{x}$-direction induced by the cutting forces and inaccuracy in the serial chains have different signs, so the total error in the loaded mode is less than in the unloaded one. This error varies from -0.6 mm to $0.1 \mathrm{~mm}$ along the trajectory and even reduced to zero when the y-coordinate is about $80 \mathrm{~mm}$. In the $\mathrm{z}$-direction, the errors caused by the assembling of non-perfect serial chains is much higher (from $0.5 \mathrm{~mm}$ to $2.1 \mathrm{~mm}$ for the Case A and from -1 mm to $1 \mathrm{~mm}$ in the Case B). To compensate these errors, the technique similar to one presented in [73] can be applied.

\section{Discussions}

The developed advanced stiffness modeling technique can be applied both to serial and parallel manipulators and has a number of advantages, some of which have not been highlighted above. Let us briefly address some of them and give guidelines for the technique application for several particular cases, which are important for engineering practice.

The first of these advantage is related to the ability to compute the stiffness matrix and the manipulator configuration under the loading both for non-singular and singular postures. For the simplified case (when the manipulator geometry is perfect), this feature has been demonstrated in our previous works $[4,10]$, where singular and non-singular stiffness matrices both for serial and parallel manipulators were derived. This paper gives the methodology to obtain similar results in more general case, when the kinematic chains are non-perfect and/or auxiliary loadings are applied. In addition, this feature allows us to detect new type of singularities (so-called elastostatic ones), that are associated with the loss of static stability under the loading. Moreover, it is 
The second advantage is associated with the ability to model manipulators of complex topology, were pure serial and strictly parallel architectures are mixed. Typical examples include heavy serial robots with gravity compensators, serial robots with parallelogram-type loops as well as parallel robots with parallelogram-based linkage. For serial robots with gravity compensators or kinematic parallelograms, the desired model can be obtained by replacing the original hybrid structure by a strictly serial one, but assuming that the equivalent joint stiffness is configuration dependent (i.e., it can be described by a non-linear function of the corresponding joint variable). More details concerning implementation of this idea can be found in [75], where a simplified stiffness model was used, without auxiliary loadings and with rigid links. Similar approach can be also applied in the case when the manipulator joints include non-linear elastic elements (which are used to compensate backlash, for instance) or it is necessary to take into account the friction impact.

For parallel manipulators with parallelogram-based linkage, the desired model can be obtained by replacing this linkage by a corresponding solid bar with two passive U-joints and an equivalent non-linear virtual spring whose stiffness depends on the internal variable (parallelogram angle). For the case of perfect kinematic chains, this idea has been implemented in [76]. It is clear that similar approach can be applied to parallelograms with non-perfect geometry or to the kinematic loops of other types (with triangles, trapezoids, pentagons and non-symmetric structure, for instance).

Non-obvious advantage of the proposed technique is its ability to integrate different error sources (manufacturing errors, assembling errors. backlash, etc.) in the elasto-static model of parallel manipulator. This feature follows from expression (60), which treats all errors in a similar way by evaluating their impact on the end-chain position/orientation, i.e. deviation from perfect case. Besides, the developed approach can be efficiently applied to the cases of both linear and non-linear errors.

Another important issue, which should not be missed in the stiffness analysis of the heavy industrial robots, is the influence of gravity forces which are associated with the own mass of the manipulator links. It is clear that these forces are applied to the link mass centers, what on the first sight contradicts to the assumption presented in Section 3.1 (see Figure 3, where all external forces are applied at the nodes corresponding to the manipulator joints). However, this difficulty can be easily overcome by presenting relevant gravity forces as equivalent pairs of parallel forces applied at adjacent joints.

And finally, this technique is rather computationally efficient, and it can be used online compliance error compensation. The main difficulty here is related to computing of static equilibrium configuration, which usually consumes the majority of computational time. However, for small position/force increments between two successive control signals update, corresponding numerical procedure converges in 1-2 iterations (if current solution from previous control step is used as an initial one for the subsequent step). This allows us to use the benefits of the developed technique for improvement of existing robot controllers without essential increasing of their computational capacity.

\section{Conclusions}

The paper presents an advanced stiffness modeling technique for parallel manipulators composed of perfect and non-perfect serial chains whose geometry differs from the nominal one. The developed technique contributes both to the stiffness modeling of serial and parallel manipulators under internal and external loadings. Particular attention has been done to enhancement of VJM-based stiffness modeling technique for the case of auxiliary loading (applied to the intermediate points). The obtained results allows us to take into account gravity forces induced by the link weights which are assumed to be applied in the intermediate points. In contrast to other works, the developed technique is able to take into account deviation of the end-platform location because of inaccuracy in the geometry of serial chains, which does not allow to assemble manipulator without internal stresses. The developed aggregation procedure combines the chain stiffness models and produces the relevant force-deflection relation, the aggregated Cartesian stiffness matrix and the reference point displacements caused by inaccuracy in kinematic chains. The developed technique can be applied to both over-constrained and under-constrained manipulators, and is suitable for the cases of both small and large deflections.

The advantages of the developed technique are illustrated by an example that deals with the over-constrained parallel manipulator of the Orthoglide architecture. It demonstrates the technique ability to evaluate the end-effector deflections caused by conventional causes (cutting forces/torques applied to the end-effector that arise while workpiece processing) and by specific ones, induced by inaccuracy in serial chains of the parallel manipulator. Relevant plots that illustrate influence of different error sources on the manipulator positioning accuracy are presented.

In future, the proposed technique will be integrated in a software toolbox that can be used for parallel manipulators of complex architecture and applied to the industrial problem of the compliance error compensation in robotic machining cells.

\section{Acknowledgements}

The work presented in this paper was partially funded by the Region "Pays de la Loire", France, by the project ANR 


\section{References}

[1] C. Shih-Feng, K. Imin, Geometrical approach to the conservative congruence transformation (CCT) for robotic stiffness control, Robotics and Automation, 2002. Proceedings. ICRA '02. IEEE International Conference on, 2002, pp. 544-549 vol.541.

[2] G. Alici, B. Shirinzadeh, Enhanced stiffness modeling, identification and characterization for robot manipulators, Robotics, IEEE Transactions on, 21 (2005) 554-564.

[3] A. Pashkevich, D. Chablat, P. Wenger, Stiffness analysis of overconstrained parallel manipulators, Mechanism and Machine Theory, 44 (2009) 966-982.

[4] A. Klimchik, A. Pashkevich, S. Caro, D. Chablat, Stiffness matrix of manipulators with passive joints: computational aspects, Robotics, IEEE Transactions on, 28 (2012) 955-958.

[5] B.-J. Yi, R.A. Freeman, Geometric analysis of antagonistic stiffness in redundantly actuated parallel mechanisms, Journal of Robotic Systems, 10 (1993) 581-603.

[6] C. Quennouelle, C.á. Gosselin, Stiffness matrix of compliant parallel mechanisms, Advances in Robot Kinematics: Analysis and Design, Springer, 2008, pp. 331-341.

[7] B.-J. Yi, R.A. Freeman, Synthesis of actively adjustable springs by antagonistic redundant actuation, ASME Transactions Journal of Dynamic Systems and Measurement Control B, 114 (1992) 454-461.

[8] D. Chakarov, Study of the antagonistic stiffness of parallel manipulators with actuation redundancy, Mechanism and Machine Theory, 39 (2004) 583-601.

[9] W. Wei, N. Simaan, Design of Planar Parallel Robots with Preloaded Flexures for Guaranteed Backlash Prevention, ASME Journal of Mechanisms and Robotics, 2 (2010).

[10] A. Pashkevich, A. Klimchik, D. Chablat, Enhanced stiffness modeling of manipulators with passive joints, Mechanism and machine theory, 46 (2011) 662-679.

[11] C. Gosselin, D. Zhang, Stiffness analysis of parallel mechanisms using a lumped model, International Journal of Robotics and Automation, 17 (2002) 17-27.

[12] J. Kövecses, J. Angeles, The stiffness matrix in elastically articulated rigid-body systems, Multibody System Dynamics, 18 (2007) 169-184.

[13] J.-P. Merlet, C. Gosselin, Parallel Mechanisms and Robots, in: B. Siciliano, O. Khatib (Eds.) Springer Handbook of Robotics, Springer Berlin Heidelberg, 2008, pp. 269-285.

[14] K. Nagai, Z. Liu, A systematic approach to stiffness analysis of parallel mechanisms, Robotics and Automation, 2008. ICRA 2008. IEEE International Conference on, IEEE, 2008, pp. 1543-1548.

[15] D. Zhang, C.M. Gosselin, Kinetostatic modeling of parallel mechanisms with a passive constraining leg and revolute actuators, Mechanism and Machine Theory, 37 (2002) 599-617.

[16] C. Corradini, J.-C. Fauroux, S. Krut, Evaluation of a 4-degree of freedom parallel manipulator stiffness, Proceedings of the 11th World Congress in Mechanisms and Machine Science, Tianjin (China), 2003

[17] Y. Li, Q. Xu, Stiffness analysis for a 3-PUU parallel kinematic machine, Mechanism and Machine Theory, 43 (2008) 186-200.

[18] J. Duffy, Statics and Kinematics with Applications to Robotics. 1996, Cambridge University Press, New York.

[19] J. Angeles, Fundamentals of robotic mechanical systems: theory, methods, and algorithms, Springer, 2007.

[20] S. Howard, M. Zefran, V. Kumar, On the $6 \times 6$ Cartesian stiffness matrix for three-dimensional motions, Mechanism and machine theory, 33 (1998) 389-408.

[21] M. Žefran, V. Kumar, A Geometrical Approach to the Study of the Cartesian Stiffness Matrix, Journal of Mechanical Design, 124 (1998) 30-38.

[22] N. Ciblak, H. Lipkin, Asymmetric Cartesian stiffness for the modeling of compliant robotic systems, Proc. 23rd Biennial ASME Mechanisms Conference, Minneapolis, MN, 1994.

[23] M. Žefran, V. Kumar, Coordinate-Free Formulation of the Cartesian Stiffness Matrix, in: J. Lenarčič, V. Parenti-Castelli (Eds.) Recent Advances in Robot Kinematics, Springer Netherlands, 1996, pp. 119-128.

[24] T. Pigoski, M. Griffis, J. Duffy, Stiffness mappings employing different frames of reference, Mechanism and machine theory, 33 (1998) 825-838.

[25] N. Ciblak, H. Lipkin, Application of Stiffness Decompositions to Synthesis by Springs, 1998 ASME Design Engineering Technical Conferences, ASME, September, 13-16, 1998, Atlanta, Georgia, 1998, pp. DETC98/MECH-5880.

[26] S.-F. Chen, I. Kao, Conservative congruence transformation for joint and Cartesian stiffness matrices of robotic hands and fingers, The International Journal of Robotics Research, 19 (2000) 835-847.

[27] S.-F. Chen, I. Kao, Simulation of conservative congruence transformation. Conservative properties in the joint and Cartesian spaces, Robotics and Automation, 2000. Proceedings. ICRA'00. IEEE International Conference on, IEEE, 2000, pp. 1283-1288.

[28] N. Ciblak, H. Lipkin, Synthesis of Cartesian stiffness for robotic applications, Robotics and Automation, 1999. Proceedings. 1999 IEEE 
Stiffness modeling for perfect and non-perfect parallel manipulators under internal and external loadings International Conference on, IEEE, 1999, pp. 2147-2152.

[29] I. Kao, C. Ngo, Properties of the Grasp Stiffness Matrix and Conservative Control Strategies, The International Journal of Robotics Research, 18 (1999) 159-167.

[30] S. Chen, I. Kao, The conservative congruence transformation of stiffness control in robotic grasping and manipulation, Proceedings of the 9th International Symposium of Robotics Research, 1999, pp. 7-14.

[31] C. Huang, W.-H. Hung, I. Kao, New conservative stiffness mapping for the Stewart-Gough platform, Robotics and Automation, 2002. Proceedings. ICRA'02. IEEE International Conference on, IEEE, 2002, pp. 823-828.

[32] B. Bouzgarrou, J. Fauroux, G. Gogu, Y. Heerah, Rigidity analysis of T3R1 parallel robot with uncoupled kinematics, Proc. of the 35th International Symposium on Robotics (ISR), Paris, France, 2004.

[33] J.E. Akin, Finite element analysis with error estimators: An introduction to the FEM and adaptive error analysis for engineering students, Butterworth-Heinemann, 2005.

[34] A. Klimchik, A. Pashkevich, D. Chablat, CAD-based approach for identification of elasto-static parameters of robotic manipulators, Finite Elements in Analysis and Design, 75 (2013) 19-30.

[35] H.C. Martin, Introduction to matrix methods of structural analysis, McGraw-Hill, 1966.

[36] S. Marie, E. Courteille, P. Maurine, Elasto-geometrical modeling and calibration of robot manipulators: Application to machining and forming applications, Mechanism and Machine Theory, 69 (2013) 13-43.

[37] D. Deblaise, X. Hernot, P. Maurine, A systematic analytical method for PKM stiffness matrix calculation, Robotics and Automation, 2006. ICRA 2006. Proceedings 2006 IEEE International Conference on, IEEE, 2006, pp. 4213-4219.

[38] D. Deblaise, Contribution à la modélisation et à l'étalonnage élasto-géométriques des manipulateurs à structure parallèle, INSA de Rennes, 2006.

[39] K. Salisbury, Active stiffness control of a manipulator in Cartesian coordinates, Decision and Control including the Symposium on Adaptive Processes, 1980 19th IEEE Conference on, IEEE, 1980, pp. 95-100.

[40] C. Gosselin, Stiffness mapping for parallel manipulators, Robotics and Automation, IEEE Transactions on, 6 (1990) $377-382$.

[41] C. Quennouelle, Modelisation geometrico-statique des mecanismes paralleles compliants, Université Laval, 2009.

[42] F. Majou, C. Gosselin, P. Wenger, D. Chablat, Parametric stiffness analysis of the Orthoglide, Mechanism and Machine Theory, 42 (2007) 296-311.

[43] D. Zhang, 2000, Kinetostatic Analysis and Optimization of Parallel and Hybrid Architectures for Machine Tools. Ph. D. thesis, Laval University.

[44] L. Lin, C. Yang, J. Lu, L. Ying, W. E, A Fast Parallel Algorithm for Selected Inversion of Structured Sparse Matrices with Application to 2D Electronic Structure Calculations, SIAM Journal on Scientific Computing, 33 (2011) 1329-1351.

[45] Y. Li, I. Kao, On the stiffness control and congruence transformation using the conservative congruence transformation (CCT), Robotics and Automation, 2001. Proceedings 2001 ICRA. IEEE International Conference on, IEEE, 2001, pp. 3937-3942.

[46] D. Zhang, F. Xi, C.M. Mechefske, S.Y.T. Lang, Analysis of parallel kinematic machine with kinetostatic modelling method, Robotics and Computer-Integrated Manufacturing, 20 (2004) 151-165.

[47] F. Pierrot, J. Fauroux, A method for modeling analytical stiffness of a lower mobility parallel manipulator, Robotics and Automation, 2005. ICRA 2005. Proceedings of the 2005 IEEE International Conference on, IEEE, 2005, pp. 3232-3237.

[48] F. Xi, D. Zhang, C.M. Mechefske, S.Y. Lang, Global kinetostatic modelling of tripod-based parallel kinematic machine, Mechanism and Machine Theory, 39 (2004) 357-377.

[49] M. Ceccarelli, G. Carbone, A stiffness analysis for CaPaMan (Cassino parallel manipulator), Mechanism and Machine Theory, 37 (2002) 427-439.

[50] O. Company, S. Krut, F. Pierrot, Modelling and preliminary design issues of a four-axis parallel machine for heavy parts handling, Proceedings of the Institution of Mechanical Engineers, Part K: Journal of Multi-body Dynamics, 216 (2002) 1-12.

[51] H.K. Arumugam, R.M. Voyles, S. Bapat, Stiffness analysis of a class of parallel mechanisms for micro-positioning applications, Intelligent Robots and Systems, 2004.(IROS 2004). Proceedings. 2004 IEEE/RSJ International Conference on, IEEE, 2004, pp. 1826-1831.

[52] E. Abele, M. Weigold, S. Rothenbücher, Modeling and identification of an industrial robot for machining applications, CIRP Annals-Manufacturing Technology, 56 (2007) 387-390.

[53] R. Vertechy, V. Parenti-Castelli, Static and stiffness analyses of a class of over-constrained parallel manipulators with legs of type US and UPS, Robotics and Automation, 2007 IEEE International Conference on, IEEE, 2007, pp. 561-567.

[54] I. Tyapin, G. Hovland, The Gantry-Tau parallel kinematic machine-kinematic and elastodynamic design optimisation, Meccanica, 46 (2011) 113-129.

[55] S. Kucuk, Energy minimization for 3-RRR fully planar parallel manipulator using particle swarm optimization, Mechanism and Machine Theory, 62 (2013) 129-149.

[56] A. Pashkevich, D. Chablat, P. Wenger, Stiffness analysis of 3-d.o.f. overconstrained translational parallel manipulators, Robotics and Automation, 2008. ICRA 2008. IEEE International Conference on, 2008, pp. 1562-1567.

[57] A. Pashkevich, A. Klimchik, S. Caro, D. Chablat, Cartesian stiffness matrix of manipulators with passive joints: Analytical approach, 

Intelligent Robots and Systems (IROS), 2011 IEEE/RSJ International Conference on, 2011, pp. 4034-4041.

[58] D. Chablat, P. Wenger, Architecture optimization of a 3-DOF translational parallel mechanism for machining applications, the orthoglide, Robotics and Automation, IEEE Transactions on, 19 (2003) 403-410.

[59] R. Rizk, N. Andreff, J.C. Fauroux, J.M. Lavest, G. Gogu, Precision Study of a Decoupled Four Degrees of Freedom Parallel Robot Including Manufacturing and Assembling Errors, in: S. Tichkiewitch, M. Tollenaere, P. Ray (Eds.) Advances in Integrated Design and Manufacturing in Mechanical Engineering II, Springer Netherlands, 2007, pp. 111-127.

[60] D. Chakarov, Study of the passive compliance of parallel manipulators, Mechanism and Machine Theory, 34 (1999) $373-389$.

[61] J.S. Przemieniecki, Theory of matrix structural analysis, Courier Dover Publications, 1985.

[62] Y. Li, S.-F. Chen, I. Kao, Stiffness control and transformation for robotic systems with coordinate and non-coordinate bases, Robotics and Automation, 2002. Proceedings. ICRA '02. IEEE International Conference on, 2002, pp. 550-555.

[63] M. Carricato, J. Duffy, V. Parenti-Castelli, Catastrophe analysis of a planar system with flexural pivots, Mechanism and Machine Theory, 37 (2002) 693-716.

[64] R. Hines, D. Marsh, J. Duffy, Catastrophe analysis of the planar two-spring mechanism, The International Journal of Robotics Research, 17 (1998) 89-101.

[65] R. Clavel, Delta, a fast robot with parallel geometry, Proceedings of the International Symposium on Industrial Robots, 1988, pp. 91-100.

[66] J.-P. Merlet, Parallel robots, Springer, 2006.

[67] J. Choi, S. Hong, W. Lee, S. Kang, M. Kim, A robot joint with variable stiffness using leaf springs, Robotics, IEEE Transactions on, 27 (2011) 229-238.

[68] J. Borras, A.M. Dollar, Static analysis of parallel robots with compliant joints for in-hand manipulation, Intelligent Robots and Systems (IROS), 2012 IEEE/RSJ International Conference on, IEEE, 2012, pp. 3086-3092.

[69] K.C. Galloway, J.E. Clark, D.E. Koditschek, Variable Stiffness Legs for Robust, Efficient, and Stable Dynamic Running, ASME, 2013.

[70] J. Guo, K.-M. Lee, Compliant joint design and flexure finger dynamic analysis using an equivalent pin model, Mechanism and Machine Theory, 70 (2013) 338-353.

[71] A. Klimchik, A. Pashkevich, D. Chablat, Stability Of Manipulator Configuration Under External Loading, Proceedings of the 11th Biennial Conference on Engineering Systems Design and Analysis, ASME, 2012, ESDA2012-82217, pp. 1-10.

[72] F.R. Gantmacher, The theory of matrices, vol. I, 1959

[73] A. Klimchik, A. Pashkevich, D. Chablat, G. Hovland, Compliance error compensation technique for parallel robots composed of non-perfect serial chains, Robotics and Computer-Integrated Manufacturing, 29 (2013) 385-393.

[74] A. Pashkevich, P. Wenger, D. Chablat, Design strategies for the geometric synthesis of Orthoglide-type mechanisms, Mechanism and Machine Theory, 40 (2005) 907-930.

[75] A. Klimchik, Y. Wu, C. Dumas, S. Caro, B. Furet, A. Pashkevich, Identification of geometrical and elastostatic parameters of heavy industrial robots, Robotics and Automation (ICRA), 2013 IEEE International Conference on, 2013, pp. 3707-3714.

[76] A. Pashkevich, A. Klimchik, S. Caro, D. Chablat, Stiffness Modelling of Parallelogram-Based Parallel Manipulators, in: D. Pisla, M. Ceccarelli, M. Husty, B. Corves (Eds.) New Trends in Mechanism Science, Springer Netherlands, 2010, pp. 675-682. 